\title{
CROSSED INTERVAL GROUPS AND OPERATIONS ON THE HOCHSCHILD COHOMOLOGY
}

\author{
MICHAEL BATANIN AND MARTIN MARKL
}

\begin{abstract}
We prove that the operad $\mathcal{B}$ of natural operations on the Hochschild cohomology has the homotopy type of the operad of singular chains on the little disks operad. To achieve this goal, we introduce crossed interval groups and show that $\mathcal{B}$ is a certain crossed interval extension of an operad $\mathcal{T}$ whose homotopy type is known. This completes the investigation of the algebraic structure on the Hochschild cochain complex that has lasted for several decades.
\end{abstract}

Dedicated to the memory of Jean-Louis Loday (12.1.1946 - 6.6. 2012)

\section{Contents}

Introduction

1. Main results 3

2. Multiplicative operads and proof of Theorem A 12

3. Crossed interval groups 17

4. A homotopy equivalence of cosimplicial totalizations. 22

5. Bar construction and proof of Theorem C 29

Appendix A. Acyclic Models Theorem for cochain functors. 32

Appendix B. Generic algebras 34

References 36

\section{INTRODUCTION}

It is well-known that, for any 'reasonable' type of algebras (where reasonable means algebras over a quadratic Koszul operad, see [19, II.3.3] or the original source [10] for the terminology), there exists the associated cohomology based on a 'standard construction.' For example, for associative algebras, the associated cohomology is the Hochschild cohomology, for Lie algebras

1991 Mathematics Subject Classification. Primary 55U10, secondary 55S05, 18 D50.

Key words and phrases. Crossed interval group, Hochschild cohomology, natural operation.

The first author gratefully acknowledges for the financial support of Scott Russel Johnson Memorial Foundation, Max Plank Institut für Mathematik and Australian Research Council (grant No. DP0558372). The second author was supported by the grant GA ČR 201/08/0397 and RVO: 67985840. 
the Chevalley-Eilenberg cohomology, for associative commutative algebras the Harrison cohomology, \&c.

There arises a fundamental problem of describing all operations acting on this associated cohomology, by which one usually means the understanding of the operad of all natural operations on the standard construction [18].

In this paper we study the operad $\mathcal{B}$ of all natural operations on the Hochschild cohomology of associative algebras. The operad $\mathcal{B}$ is the totalization of a certain coloured operad $B$ with the set of colours the natural numbers $\mathbb{N}$. We recall the intuitive definition of $B$ from [18, Section 7] in terms of 'elementary' natural operations, and formulate also a categorical, 'coordinate-free' definition which gives a precise meaning to the naturality of operations. This answers a remark of Kontsevich and Soibelman [14, page 26] concerning the nonfunctoriality of the Hochschild complex on an earlier attempt by McClure and Smith [22, page 2] to define natural operations. We then prove, in Theorem $\mathbf{A}$, that the two definitions are equivalent.

In connection with the Deligne conjecture [5], various suboperads of $\mathcal{B}$ have been studied and several results about their homotopy types were proved [3, 12, 14, 22, 23, 25]. There is for instance a differential graded suboperad $\mathcal{T}$ of the operad $\mathcal{B}$ whose topological version was introduced in [25]. The homotopy type of $\mathcal{T}$ was established in [1] as that of the operad of singular chains on the little discs operad. The homotopy type of a topological version of $\mathcal{T}$ was studied earlier in [23].

The starting point of this work therefore concerned the inclusion $\mathcal{T} \hookrightarrow \mathcal{B}$. It turns out that the operad $\mathcal{B}$ is, in a certain sense, freely generated by $\mathcal{T}$. More precisely, $\mathcal{B}$ is the free $\mathcal{J} S$ module generated by the $\mathcal{J}$-module $\mathcal{T}, \mathcal{B}=F_{\mathcal{S}}(\mathcal{T})$, see Proposition 5.1. Here $\mathcal{J} S$ is an analog of the symmetric category $\Delta S$ introduced in [7] (but see also [15]), with the simplicial category $\Delta$ replaced by Joyal's category $\mathcal{J}$ of intervals [11]. Theorem 4.4 says that the functor $F_{\mathcal{S}}(-)$ preserves the homotopy type. Combining this feature with the observations in the previous paragraph we obtain Theorem B, claiming that the operad $\mathcal{B}$ has the homotopy type of the operad of singular chains on the little disks operad $\mathcal{D}$ with the inverted grading. To our best knowledge, this is the first complete description of the homotopy type of the operad $\mathcal{B}$, compare also the introduction to [18].

Besides the suboperad $\mathcal{T}$ of $\mathcal{B}$ mentioned above, one has the important McClure-Smith operad of braces $\mathcal{B} r$ and the non-unital (resp. normalized) versions $\widehat{\mathcal{B}}, \widehat{\mathcal{T}}, \widehat{\mathcal{B} r}$ (resp. $\operatorname{Nor}(\mathcal{T}), \operatorname{Nor}(\mathcal{B})$, $\operatorname{Nor}(\mathcal{B} r)$ ) of these operads. In Theorem $\mathbf{C}$ we relate these operads and describe their homotopy types.

\footnotetext{
${ }^{1}$ Since this introduction was not intended as an account on the history of the Deligne conjecture, the list is necessarily incomplete and we apologize to everyone whose work we did not mention here.
} 
Conventions. Algebraic objects in this paper live in the category of abelian groups, i.e. are modules over the ring $\mathbb{Z}$ of integers. Therefore, for instance, $\otimes$ denotes the tensor product over $\mathbb{Z}$. All operads in this paper are symmetric, i.e. with right actions of the symmetric groups on their components. The grading (homological on cohomological) will sometimes be indicated by $*$ in the sub- or superscript, the simplicial and cosimplicial degrees by $\bullet$. The paper makes ample use of the material of [1] and [2].

Acknowledgment. Both authors of the paper were deeply shocked by the tragic death of JeanLouis Loday in June 2012. We would like to dedicate this paper to the memory of our friend whose mathematical talent and brilliant personality will always be remembered and admired. This paper would never be published without his kind encouragement and support. We would also like to express our thanks to him and Zig Fiedorowicz for their readiness to share their expertise and ideas on crossed simplicial groups with us.

Also the remarks of the referee and editors lead to substantial improvement of the paper.

\section{MAin RESUlts}

In this section, which can be read independently on the rest of the paper, we formulate our main results, Theorems A, B and C. Although Theorem B follows from Theorem C, we decided to state this important result separately, since Theorem $\mathrm{C}$ requires more background material and we did not want to stretch the reader's patience more than necessary.

We open this section by defining the dg-operad $\mathcal{B}=\{\mathcal{B}(n)\}_{n \geq 0}(\mathcal{B}$ abbreviating the 'big') of all natural multilinear operations on the Hochschild cochain complex $C^{*}(A ; A)=\operatorname{Lin}\left(A^{\otimes *}, A\right)$ of an associative algebra $A$ with coefficients in itself and describe its homotopy type. In the second half we discuss various suboperads and variants of $\mathcal{B}$ including the famous operad of braces. We finally formulate a theorem relating these operads and describing their homotopy types.

The dg-operad $\mathcal{B}$ is the totalization of a certain $\mathbb{N}$-coloured operad $B$ whose component $B_{k_{1}, \ldots, k_{n}}^{l}$ consists of natural operations of type $\left(l ; k_{l}, \ldots, k_{n}\right), l, k_{l}, \ldots, k_{n} \geq 0$. There are several equivalent ways to say what a 'natural operation' is. There are some 'elementary' operations on the Hochschild complex, see Definition 1.1, which are meaningful for any associative unital algebra. One can define a coloured operad generated by compositions of linear combinations of these operations, see Proposition 1.3. We call this definition intuitive.

Another, 'coordinate-free' Definition 1.5, requires that suitably defined natural operations must act on the endomorphisms operads of monoids $M$ in an arbitrary additive symmetric monoidal category $V$. The Hochschild cochain complex $C^{*}(A ; A)$ is clearly a special case of such an endomorphism operad with $M=A$ and $V=\mathcal{A} b$, the category of abelian groups. The naturality of operations in the second approach has a standard categorical content and does 
not require introducing any kind of 'elementary' operations. By Theorem A on page 6 both definitions give isomorphic results.

We start with the intuitive definition of the operad $B$. Let $A$ be a unital associative algebra. A natural operation in the sense of [18] is a linear combination of compositions of the following 'elementary' operations:

(a) The insertion $\circ_{i}: C^{k}(A ; A) \otimes C^{l}(A ; A) \rightarrow C^{k+l-1}(A ; A)$ given, for $k, l \geq 0$ and $1 \leq i \leq k$, by the formula

$$
\circ_{i}(f, g)\left(a_{1}, \ldots, a_{k+l-1}\right):=f\left(a_{0}, \ldots, a_{i-1}, g\left(a_{i}, \ldots, a_{i+l-1}\right), a_{i+l}, \ldots, a_{k+l-1}\right) .
$$

(b) Let $\mu: A \otimes A \rightarrow A$ be the associative product, $i d: A \rightarrow A$ the identity and $1 \in A$ the unit. Then elementary operations are also the 'constants' $\mu \in C^{2}(A ; A)$, id $\in C^{1}(A ; A)$ and $1 \in C^{0}(A ; A)$.

(c) The assignment $f \mapsto f \sigma$ permuting the inputs of a cochain $f \in C^{k}(A ; A)$ according to a permutation $\sigma \in S_{k}$ is an elementary operation.

Definition 1.1. Let $B(A)_{k_{1}, \ldots, k_{n}}^{l}$ denote, for $l, k_{q}, \ldots, k_{n} \geq 0$, the abelian group of all natural, in the above sense, operations

$$
O: C^{k_{1}}(A ; A) \otimes \cdots \otimes C^{k_{n}}(A ; A) \rightarrow C^{l}(A ; A) .
$$

The spaces $B(A)_{k_{1}, \ldots, k_{n}}^{l}$ clearly form an $\mathbb{N}$-coloured suboperad $B(A)$ of the endomorphism operad of the $\mathbb{N}$-coloured collection $\left\{C^{n}(A ; A)\right\}_{n \geq 0}$.

It follows from definition that elements of $B(A)_{k_{1}, \ldots, k_{n}}^{l}$ can be represented by linear combinations of $\left(l ; k_{1}, \ldots, k_{n}\right)$-trees in the sense of Definition 1.2 below in which, as usual, the arity of a vertex of a rooted tree is the number of its input edges and the legs are the input edges of a tree, see [19, II.1.5] for the terminology.

Definition 1.2. Let $l, k_{1}, \ldots, k_{n}$ be non-negative integers. An $\left(l ; k_{1}, \ldots, k_{n}\right)$-tree is a planar tree with legs labeled by $1, \ldots, l$ and three types of vertices:

(a) 'white' vertices of arities $k_{1}, \ldots, k_{n}$ labeled by $1, \ldots, n$,

(b) 'black' vertices of arities $\geq 2$ and

(c) 'special' vertices of arity 0 (no input edges).

We moreover require that there are no edges connecting two black vertices or a black vertex with a special vertex. For $n=0$ we allow also the exceptional trees $\mid$ and $\downarrow$ with no internal vertices.

We call an internal edge whose initial vertex is special a stub (also called, in [14, a tail). It follows from definition that the terminal vertex of a stub is white; the exceptional tree $\downarrow$ is not a stub. An example of an $\left(l ; k_{1}, \ldots, k_{n}\right)$-tree is given in Figure 1 . 


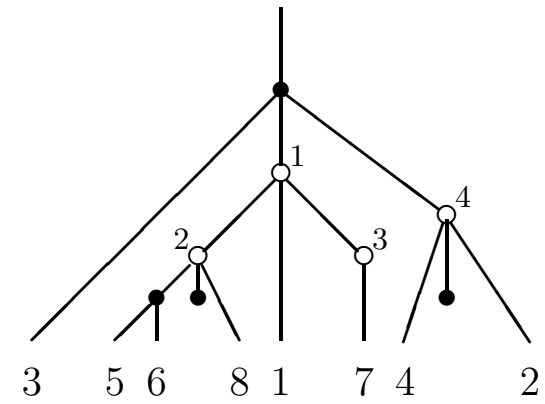

Figure 1. An $(8 ; 3,3,1,3)$-tree representing an operation in $B_{3,3,1,3}^{8}(A)$. It has 4 white vertices, 2 black vertices and 2 stubs. We use the convention that directed edges point upwards.

An $\left(l ; k_{1}, \ldots, k_{n}\right)$-tree $T$ determines the natural operation $O_{T} \in B(A)_{k_{1}, \ldots, k_{n}}^{l}$ whose action on $C^{*}(A ; A)$ is given by decorating, for each $1 \leq i \leq n$, the $i$ th white vertex by $f_{i} \in C^{k_{i}}(A ; A)$, the black vertices by the iterated multiplications, the special vertices by the unit 1 , and performing the composition along the tree. For instance, the tree in Figure 1 represents the operation

$$
O_{T}\left(f_{1}, f_{2}, f_{3}, f_{4}\right)\left(a_{1}, \ldots, a_{8}\right):=a_{3} f_{1}\left(f_{2}\left(a_{5} a_{6}, 1, a_{8}\right), a_{1}, f_{3}\left(a_{7}\right)\right) f_{4}\left(a_{4}, 1, a_{2}\right)
$$

where, as usual, we omit the symbol for the iteration of the associative multiplication $\mu$. The exceptional $(1 ;)$-tree I represents the identity $i d \in C^{1}(A ; A)$ and the $(0 ;)$-tree $\downarrow$ the unit $1 \in C^{0}(A ; A)$.

Notation. For each $l, k_{1}, \ldots, k_{n} \geq 0$ denote by $B_{k_{1}, \ldots, k_{n}}^{l}$ the free abelian group spanned by all $\left(l ; k_{1}, \ldots, k_{n}\right)$-trees. The correspondence $T \mapsto O_{T}$ defines, for each associative algebra $A$, a linear epimorphism $\omega_{A}: B_{k_{1}, \ldots, k_{n}}^{l} \rightarrow B(A)_{k_{1}, \ldots, k_{n}}^{l}$.

Let $T^{\prime}$ be an $\left(l^{\prime} ; k_{1}^{\prime}, \ldots, k_{n}^{\prime}\right)$-tree, $T^{\prime \prime}$ an $\left(l^{\prime \prime} ; k_{1}^{\prime \prime}, \ldots, k_{m}^{\prime \prime}\right)$-tree and assume that $l^{\prime \prime}=k_{i}^{\prime}$ for some $1 \leq i \leq n$. The $i$ th vertex insertion assigns to $T^{\prime}$ and $T^{\prime \prime}$ the tree $T^{\prime} \circ_{i} T^{\prime \prime}$ obtained by replacing the white vertex of $T^{\prime}$ labelled $i$ by $T^{\prime \prime}$. It may happen that this replacement creates edges connecting black vertices. In that case it is followed by collapsing these edges. The above construction extends into a linear operation

$$
\circ_{i}: B_{k_{1}^{\prime}, \ldots, k_{n}^{\prime}}^{l^{\prime}} \otimes B_{k_{1}^{\prime \prime}, \ldots, k_{m}^{\prime \prime}}^{l^{\prime \prime}} \rightarrow B_{k_{1}^{\prime}, \ldots, k_{i-1}^{\prime}, k_{1}^{\prime \prime}, \ldots, k_{m}^{\prime \prime}, k_{i+1}^{\prime}, \ldots, k_{n}^{\prime}}^{l^{\prime}}, 1 \leq i \leq n, l^{\prime \prime}=k_{i}^{\prime} .
$$

Proposition 1.3. The spaces $B_{k_{1}, \ldots, k_{n}}^{l}$ assemble into an $\mathbb{N}$-coloured operad $B$ with the operadic composition given by the vertex insertion and the symmetric group relabeling the white vertices. With this operad structure, the maps $\omega_{A}: B_{k_{1}, \ldots, k_{n}}^{l} \rightarrow B(A)_{k_{1}, \ldots, k_{n}}^{l}$ form an epimorphism $\omega_{A}$ : $B \rightarrow B(A)$ of $\mathbb{N}$-coloured operads.

Proof. A direct verification.

Definition 1.4. A unital associative algebra $A$ is generic if the map $\omega_{A}: B \rightarrow B(A)$ is an isomorphism. 
Theorem B.1 proved in Appendix B states that free associative algebras generated by countably many generators are generic, so generic algebras exist. The operad $B$ can thus be equivalently defined as the operad of elementary operations acting on the Hochschild complex of a generic algebra. This point of view will be used in our description of the isomorphism between the underlying category of the coloured operad $B$ and the linearization of the category $(\mathcal{J} S)^{\text {op }}$ given in Section 5 .

Let us proceed to the 'coordinate-free' definition of the 'big' operad. Denote by SymCat. the category whose objects are pairs $(V, M)$ of an additive strict symmetric monoidal category $V$ and a unital monoid $M$ in $V$. Morphisms are additive strict symmetric monoidal functors preserving distinguished monoids. It is interesting to note that the initial object of SymCat॰ is the category $(\Delta S)_{\text {alg }}$ recalled on page 19, with 1 as the distinguished monoid.

Definition 1.5. Let $\check{B}_{k_{1}, \ldots, k_{n}}^{l}$ be, for $l, k_{1}, \ldots, k_{n} \geq 0$, the abelian group of families of linear maps

$$
\alpha_{(V, M)}: \underline{V}\left(M^{\otimes k_{1}}, M\right) \otimes \cdots \otimes \underline{V}\left(M^{\otimes k_{n}}, M\right) \rightarrow \underline{V}\left(M^{\otimes l}, M\right)
$$

indexed by objects $(V, M) \in$ SymCat. which are natural in the sense that, for any morphism $F:(V, M) \rightarrow(W, N)$, the diagram

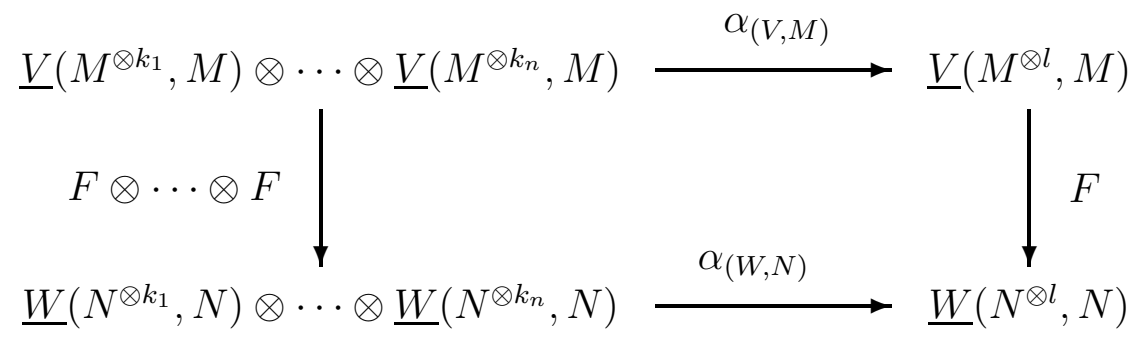

commutes. The spaces $\check{B}_{k_{1}, \ldots, k_{n}}^{l}$ clearly assemble into a coloured operad $\check{B}$ in the category $\mathcal{A} b$ of abelian groups, with natural numbers $\mathbb{N}=\{0,1,2, \ldots\}$ as its set of colors.

The following first main result of our paper is proved in Section 2 .

Theorem A. The $\mathbb{N}$-coloured operads $\check{B}$ and $B$ are canonically isomorphic.

Remark 1.6. The isomorphism $\check{B} \cong B$ is a particular feature of the (multi)linearity implied by the $\mathcal{A} b$-enrichment used in this paper. The operad $\check{B}$ is, for general enrichments, bigger than $B$. For instance, in the Cartesian situation it contains also the projections

$$
\pi_{(V, M)}^{i}: \underline{V}\left(M^{\otimes k_{1}}, M\right) \times \cdots \times \underline{V}\left(M^{\otimes k_{n}}, M\right) \rightarrow \underline{V}\left(M^{\otimes k_{i}}, M\right), 1 \leq i \leq n .
$$

\footnotetext{
${ }^{2}$ Using other types of symmetric monoidal categories and symmetric monoidal functors leads, however, to the same result.
}

[July 2012] 
The operads $B$ and $\check{B}$ have obvious nonsymmetric analogs. More precisely, let $\check{T}$ be the $\mathbb{N}$ coloured operad obtained by taking in Definition 1.5 instead of SymCat. the category of pairs $(V, M)$ consisting of a nonsymmetric monoidal category $V$ and a unital monoid $M$ in $V$. Likewise, let $T$ be the result of removing (c) from the list of elementary operations used in Definition 1.1. 1 . The following analog of Theorem A holds.

Theorem 1.7. The $\mathbb{N}$-coloured operads $T$ and $\check{T}$ are canonically isomorphic.

The operad $B$ has, as each $\mathbb{N}$-colored operad in $\mathcal{A} b$, its underlying category $\mathcal{U}(B)$ enriched in $\mathcal{A} b$, whose objects are natural numbers and the enriched Hom-sets are $\mathcal{U}(B)(k, l):=B_{k}^{l}$. The $\circ_{i}$-operation of (1) taken with $i=n=m=1, l^{\prime}=l, l^{\prime \prime}=k_{1}^{\prime}=k$ and $k_{1}^{\prime \prime}=m$, i.e. a map $B_{k}^{l} \otimes B_{m}^{k} \rightarrow B_{m}^{l}$, gives the categorial composition.

Recall that the simplicial category $\Delta$ has objects the finite ordinals $[n]=\{0, \ldots, n\}, n \geq 0$. Morphism of $\Delta$ are generated by the face maps $\delta_{i}:[n-1] \rightarrow[n]$ (misses $i$ ) and the degeneracy maps $\sigma_{i}:[n+1] \rightarrow[n]$ (hits $i$ twice), $i=0, \ldots, n$. There is a natural functor $u: \Delta \rightarrow \mathcal{U}(B)$ such that $u([n]):=n, u\left(\delta_{i}\right) \in B_{n-1}^{n}$ is defined as

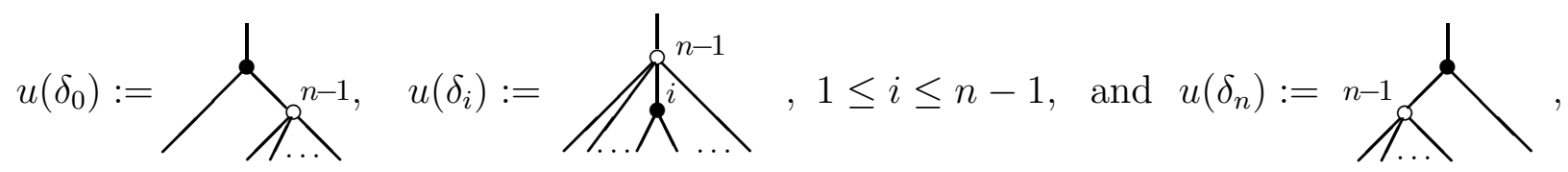

and $u\left(\sigma_{i}\right) \in B_{n+1}^{n}$ as

$$
u\left(\sigma_{i}\right):=\overbrace{\ldots}^{n+1}, 0 \leq i \leq n .
$$

In the displays, $n-1$, resp. $n+1$, denotes the arity of the white vertex and $i$ indicates its $i$ th input. The above trees obviously act on the Hochschild complex of an associative algebra via the standard cosimplicial structure.

It follows from general properties of coloured operads that $B_{k}^{l}$ acts on $B_{k_{1}, \ldots, k_{n}}^{l}$ covariantly on the upper index and contravariantly on each lower index. Therefore, the spaces $B_{k_{1}, \ldots, k_{n}}^{l}$ assemble into a functor

$$
B_{\bullet_{1}, \ldots, \bullet_{n}}^{\bullet}: \Delta \times\left(\Delta^{\mathrm{op}}\right)^{n} \rightarrow \mathcal{A} b .
$$

We can totalize the functor $B_{\bullet_{1}, \ldots, \bullet_{n}}^{\bullet}$ (i.e. we apply the cosimplicial Tot with respect to the upper index and multisimplicial Tot with respect to the lower indices). That is, for any $n \geq 0$ we put

$$
\mathcal{B}^{*}(n):=\overline{\operatorname{Tot}}\left(\underline{\operatorname{Tot}} B_{\bullet_{1}, \ldots, \bullet_{n}}^{\bullet}\right)=\prod_{l-\left(k_{1}+\cdots+k_{n}\right)=*} B_{k_{1}, \ldots, k_{n}}^{l}
$$

\footnotetext{
${ }^{3}$ This operad was first described in 25, Section 3]. Still another presentation of $T$ can be found in 2 , Proposition 4.10] or in the proof of [1, Proposition 2.14].

${ }^{4}$ The cosimplicial totalization is recalled in Section 4 , the (multi)simplicial totalization is the standard one.
} 


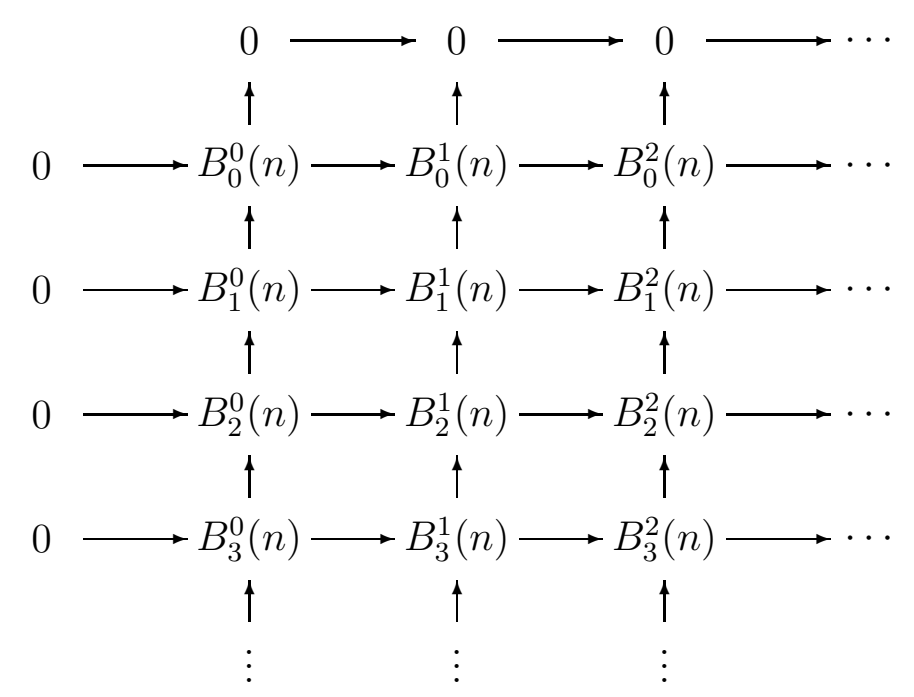

FiguRE 2. The structure of the big operad $\mathcal{B}$. In the above diagram, $B_{k}^{m}(n):=$ $\prod_{k_{1}+\cdots+k_{n}=k} B_{k_{1}, \ldots, k_{n}}^{m}$. The vertical arrows are the simplicial differentials $\partial$ and the horizontal arrows are the cosimplicial differentials $\delta$.

The degree +1 differential $d: \mathcal{B}^{*}(n) \rightarrow \mathcal{B}^{*+1}(n)$ is thus given by $d:=\delta-\left(\partial_{1}+\cdots+\partial_{n}\right)$, where $\delta$ (resp. each $\partial_{i}, 1 \leq i \leq n$ ) is induced from the corresponding cosimplicial (resp. simplicial) structure.

It is not hard to observe (see the appendix of [2] for a formal categorical proof) that the collection $\mathcal{B}=\left\{\mathcal{B}^{*}(n)\right\}_{n \geq 0}$ is a dg operad which acts on the cosimplicial totalization $C^{*}(A ; A)=$ $\overline{\operatorname{Tot}}\left(C^{\bullet}(A ; A)\right)$ of the Hochschild cochains with the standard structure of the cosimplicial abelian group. We remind the reader that, according to our conventions, $C^{*}(A ; A)$ denotes the Hochschild cochain complex while $C^{\bullet}(A ; A)$ the cosimplicial abelian group of the Hochschild cochains.

The structure of the operad $\mathcal{B}$ is visualized in Figure 2. The degree $m$-piece of $\mathcal{B}(n)$ is the direct product, not the direct sum, of elements on the diagonal $p+q=m$. Therefore the usual spectral sequence arguments do not apply. For instance, it can be shown that all rows in Figure 2 are acyclic, but $\mathcal{B}(n)$ is not acyclic! We have

Theorem B. The big dg-operad $\mathcal{B}$ of all natural operations on the Hochschild complex has the homotopy type of the dg-operad $C_{-*}(\mathcal{D})$ of singular chains on the little disks operad $\mathcal{D}$ with the inverted grading.

Theorem B is a consequence of Theorem $\mathrm{C}$ formulated at the end of this section.

Variants 1.8. An important suboperad of $\mathcal{B}$ is the suboperad $\widehat{\mathcal{B}}$ spanned by trees without stubs and without the exceptional tree $\boldsymbol{b}$. The operad $\widehat{\mathcal{B}}$ is the operad of all natural multilinear operations on the Hochschild complex of a non-unital associative algebra. It is generated by natural operations (a)-(c) above but without the unit $1 \in C^{0}(A ; A)$ in (b). 
As we already know, the arity $n$-component $\mathcal{B}(n)$ of the operad $\mathcal{B}$ is, for each $n \geq 0$, an $n$ times simplicial abelian group. One can therefore consider its normalization $\operatorname{Nor}(\mathcal{B})(n)$, i.e. the quotient of $\mathcal{B}(n)$ by images of the simplicial degeneracies. Since the simplicial degeneracies of the $i$ th simplicial structure, $1 \leq i \leq n$, act by growing stubs from the white vertex labelled $i$, we see that, as dg-abelian groups,

$$
\operatorname{Nor}(\mathcal{B})(n) \cong \begin{cases}\widehat{\mathcal{B}}(n), & \text { for } n \geq 1, \text { and } \\ \mathcal{B}(0), & \text { for } n=0 .\end{cases}
$$

The space $\widehat{\mathcal{B}}(0)$ is a proper subset of $\operatorname{Nor}(\mathcal{B})(0)$ as the latter contains also the span of the 'exceptional tree' $\boldsymbol{d}$. It is not difficult to see that the componentwise normalizations $\{\operatorname{Nor}(\mathcal{B})(n)\}_{n \geq 0}$ assemble into an operad $\operatorname{Nor}(\mathcal{B})$. One may also identify $\operatorname{Nor}(\mathcal{B})$ with the subcollection of $\mathcal{B}$ spanned by trees without stubs, but keeping in mind that $\operatorname{Nor}(\mathcal{B})$ is not a suboperad of $\mathcal{B}$.

The operad $\widehat{\mathcal{B}}$ is the same as the operad introduced, under the name $\mathcal{B}_{\mathcal{A s s}}$, in $[1 \mathbb{1} \|$. In the notation of that paper, $\mathcal{B}$ would be denoted by $\mathcal{B}_{U \mathcal{A} s s}$. It was shown by direct calculations, in 18 , Example 12], that $\mathcal{B}_{\mathcal{A} s s}(0) \cong \widehat{\mathcal{B}}(0)$ are acyclic dg-abelian groups.

While $\widehat{\mathcal{B}}$ acts on the Hochschild complex of a non-unital algebra, the operad Nor $(\mathcal{B})$ acts on the normalized Hochschild complex of a unital algebra. One has the chain of operad maps

$$
\widehat{\mathcal{B}} \stackrel{\iota}{\hookrightarrow} \mathcal{B} \stackrel{\pi}{\rightarrow} \operatorname{Nor}(\mathcal{B})
$$

in which the projection $\pi$ is a weak equivalence and the components $\pi \iota(n)$ of the composition $\pi \iota$ are isomorphisms for each $n \geq 1$. If $\mathfrak{U}$ denotes the functor that replaces the arity zero component of a dg-operad by the trivial abelian group, then $\mathfrak{U}(\pi \iota)$ is clearly a dg-operad isomorphism $\mathfrak{U}(\widehat{\mathcal{B}}) \cong \mathfrak{U}(\operatorname{Nor}(\mathcal{B}))$.

The operad $\mathcal{T}$. Totalizing the operad $T$ of Theorem 1.7 in the same way as $B$ we produce a suboperad $\mathcal{T}$ of $\mathcal{B}$ whose arity- $n$ piece equals

$$
\mathcal{T}^{*}(n):=\overline{\operatorname{Tot}}\left(\underline{\operatorname{Tot}} T_{\bullet_{1}, \ldots, \bullet_{n}}^{\bullet}\right)^{*}=\prod_{l-\left(k_{1}+\cdots+k_{n}\right)=*} T_{k_{1}, \ldots, k_{n}}^{l},
$$

where operations in $T_{k_{1}, \ldots, k_{n}}^{l}$ are represented by linear combinations of unlabeled $\left(l ; k_{1}, \ldots, k_{n}\right)$ trees, that is, planar trees as in Definition 1.2 but without the labels of the legs. The inclusion $T_{k_{1}, \ldots, k_{n}}^{l} \hookrightarrow B_{k_{1}, \ldots, k_{n}}^{l}$ is realized by labeling the legs of an unlabeled tree counterclockwise in the orientation given by the planar embedding. The operad $\mathcal{T}$ is a chain version of the operad considered in [25, Section 3].

There are finally the operad $\widehat{\mathcal{T}}:=\widehat{\mathcal{B}} \cap \mathcal{T}$ generated by unlabeled trees without stubs and without $\downarrow$, and the normalized quotient $\operatorname{Nor}(\mathcal{T})$ of $\mathcal{T}$. As before, one has the diagram

$$
T N \stackrel{\iota}{\hookrightarrow} \mathcal{T} \stackrel{\pi}{\rightarrow} \operatorname{Nor}(\mathcal{T})
$$

with the properties analogous to that of (5). 
Operads of braces. There is another very important suboperad $\mathcal{B} r$ of $\mathcal{B}$ generated by braces, cup-products and the unit. More precisely, $\mathcal{B} r$ is the suboperad of the big operad $\mathcal{B}$ generated by the following operations.

(a) The cup product $C^{*}(A ; A) \otimes C^{*}(A ; A) \rightarrow C^{*}(A ; A)$ given, for $f \in C^{k}(A ; A)$ and $g \in$ $C^{l}(A ; A)$, by

$$
(f \cup g)\left(a_{1}, \ldots, a_{k+l}\right):=f\left(a_{1}, \ldots, a_{k}\right) g\left(a_{k+1}, \ldots, a_{k+l}\right) .
$$

(b) The constant $1 \in C^{0}(A ; A)$.

(c) The braces $-\{-, \ldots,-\}: C^{*}(A ; A)^{\otimes n} \rightarrow C^{*}(A ; A), n \geq 2$, given by

$$
f\left\{g_{2}, \ldots, g_{n}\right\}:=\sum(-1)^{\epsilon} f\left(i d, \ldots, i d, g_{2}, i d, \ldots, i d, g_{n}, i d, \ldots, i d\right),
$$

where $i d$ is the identity map of $A$, the summation runs over all possible substitutions of $g_{2}, \ldots, g_{n}$ (in that order) into $f$ and

$$
\epsilon:=\sum_{j=2}^{n}\left(\operatorname{deg}\left(g_{i}\right)-1\right) t_{j},
$$

in which $t_{j}$ is the total number of inputs in front of $g_{j}$.

The brace operad has also its non-unital version $\widehat{\mathcal{B} r}:=\widehat{\mathcal{B}} \cap \mathcal{B} r$ generated by elementary operations (a) and (c). One can verify that both $\mathcal{B} r$ and $\widehat{\mathcal{B} r}$ are indeed dg-suboperads of $\mathcal{B}$, see [2, 22].

To complete the picture, we denote by $\operatorname{Nor}(\mathcal{B} r) \subset \operatorname{Nor}(\mathcal{B})$ the image of $\mathcal{B} r$ under the projection $\mathcal{B} \rightarrow \operatorname{Nor}(\mathcal{B})$. One has again an analog $\widehat{\mathcal{B} r} \stackrel{\iota}{\hookrightarrow} \mathcal{B} r \stackrel{\pi}{\rightarrow} \operatorname{Nor}(\mathcal{B} r)$ of (5).

It turns out that the piece $\mathcal{B} r(n)$ of the operad $\mathcal{B} r$ is, for each $n \geq 0$, the unnormalized chain complex associated to the $n$-times simplicial abelian group $T\left(\bullet, \ldots, \bullet_{n} ; 0\right):\left(\Delta^{\mathrm{op}}\right)^{\times n} \rightarrow \mathcal{A} b$, see [2, Section 3.1] for details. The chain complex $\operatorname{Nor}(\mathcal{B} r)(n)$ is then the simplicial normalization of $T\left(\bullet_{1}, \ldots, \bullet_{n} ; 0\right)$, so the map $\mathcal{B} r \stackrel{\pi}{\rightarrow} \operatorname{Nor}(\mathcal{B} r)$ is a weak equivalence as in the previous cases.

Remark. The operads $\operatorname{Nor}(\mathcal{B} r)$ and $\widehat{\mathcal{B} r}$ were first introduced by McClure and Smith [22] under the names $\mathcal{H}$ and $\mathcal{H}^{\prime}$, respectively.

Recall that $\mathfrak{U}$ is the functor that replaces the arity zero component of a dg-operad by the trivial abelian group. The last main result of this paper is:

Theorem C. The operads introduced above can be organized into the diagram in Figure 3 in which:

(1) Operads in the two upper triangles have the chain homotopy type of the operad $C_{-*}(\mathcal{D})$ of singular chains on the little disks operad $\mathcal{D}$ with the inverted grading.

(2) all morphisms between vertices of the two upper triangles are weak equivalences,

(3) operads in the bottom triangle of Figure 3 have the chain homotopy type of the operad $C_{-*}(\mathcal{D})$ with the component of arity 0 replaced by the trivial abelian group, and 


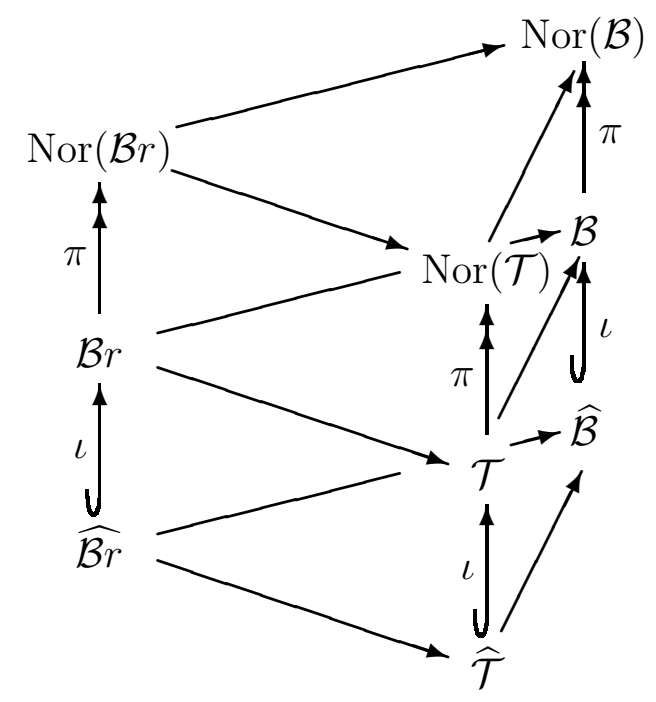

Figure 3. Operads of natural operations and their maps. The horizontal maps are inclusions.

(4) all morphisms in Figure 3 become weak equivalences after the application of the functor $\mathfrak{U}$.

Theorem $\mathrm{C}$ is proved in Section 5 .

\section{Plan for the proofs.}

Section 2. To prove Theorem A that states that both the intuitive and categorical definitions of the $\mathbb{N}$-coloured operad $B$ lead to isomorphic results, we introduce, in Definition 2.5, an auxiliary $\mathbb{N}$-coloured operad $\mathcal{N}$ at of natural operations acting on multiplicative operads with unit. Theorem A will then follow from Propositions 2.6 and 2.9 claiming that both definitions of $B$ give operads isomorphic to $\mathcal{N} a t$.

Section 3. We introduce crossed interval groups and describe our main example, the crossed interval group $\mathcal{S}$ with the underlying category $\mathcal{J} S$. For a crossed interval group $\mathcal{H}$, we define $\mathcal{H}$-objects in a category $\mathcal{C}$ and describe, in Lemma 3.12, the left adjoint $F_{\mathcal{H}}$ to the restriction functor from the category of $\mathcal{H}$-objects in $\mathcal{C}$ to the category of cosimplicial objects in $\mathcal{C}$.

Section 4 . This section is devoted to the particular case of $\mathcal{S}$-objects in the category Chain of chain complexes. We use an acyclic-models-type argument developed in Appendix \& to prove, in Theorem 4.4, that the functor $F_{\mathcal{S}}$ preserves the homotopy type.

Section 5. We show that the pieces of the coloured operad $B$ assemble into a functor from $(\mathcal{J} S)^{\text {op }} \times(\mathcal{J} S)^{\times n}$ to the category of abelian groups. By Proposition 5.1, this functor is the result of the application of the functor $F_{\mathcal{S}}$ to the pieces of a coloured operad whose homotopy type is known. This, along with Theorem 4.4 and a couple of standard arguments, implies Theorem C.

Appendix A. We introduce a theory of acyclic models for functors with values in cochain complexes. The main result, Theorem A.4, is a dual version of the classical (chain) Acyclic Models Theorem. 
Appendix B. The concept of generic algebras introduced in Section 1 allows to view elements of the operad $B$ as multilinear operations on the Hochschild complex of a concrete algebra. In this part of the appendix we show that free associative unital algebras generated by countably many generators are generic.

\section{Multiplicative operads AND Proof of Theorem A}

The aim of this section is to prove Theorem A and necessary auxiliary results. We start by analyzing the structure of the operad $B$, using mostly the material taken from [2] and [18]. We then prove, in Proposition 2.6, that the operad $\check{B}$ is isomorphic to an 'auxiliary' operad $\mathcal{N} a t$ of natural operations acting on multiplicative operads with unit. Finally, we show in Proposition 2.9 that $B \cong \mathcal{N}$ at. This will imply Theorem A.

The identification of $B_{k_{1}, \ldots, k_{n}}^{l}$ with the span of the set of $\left(l ; k_{1}, \ldots, k_{n}\right)$-trees offers another description of the pieces of the operad $B$. Denote by $\mathcal{F r}$ the quotient

$$
\mathcal{F} r:=\frac{\Gamma\left(o_{1}, \ldots, o_{n}, \mu, e\right)}{\left(\mu \circ_{1} \mu=\mu \circ_{2} \mu, \mu \circ_{1} e=\mu \circ_{2} e=i d\right)},
$$

of the free operad $\Gamma\left(o_{1}, \ldots, o_{n}, \mu, e\right)$ generated by $n$ operations $o_{i} \in \mathcal{F} r\left(k_{i}\right)$ of arities $k_{i}, 1 \leq i \leq n$, one operation $\mu$ of arity 2 and one operation $e$ of arity 0 , modulo the ideal generated by the associativity $\mu \circ_{1} \mu=\mu \circ_{2} \mu$ and unitality $\mu \circ_{1} e=\mu \circ_{2} e=i d$ of $\mu$.

Let $\mathcal{F} r_{1, \ldots, 1}(l)$ be the subspace of elements of $\mathcal{F} r(l)$ containing each generator $o_{1}, \ldots, o_{n}$ precisely once. The structure theorem for free operads [17, Section 4] immediately gives:

Proposition 2.1. For each $l, k_{1}, \ldots, k_{n} \geq 0$ and $\mathcal{F} r_{1, \ldots, 1}(l)$ as above, one has the canonical isomorphism of abelian groups

$$
B_{k_{1}, \ldots, k_{n}}^{l} \cong \mathcal{F} r_{1, \ldots, 1}(l)
$$

Let $U \mathcal{A}$ ss be the operad for unital associative algebras.

Definition 2.2. 0 A multiplicative operad with unit is an operad $\mathcal{O}$ together with a morphism $p: U \mathcal{A} s s \rightarrow \mathcal{O}$, i.e. an operad under $U \mathcal{A} s s$. Equivalently, $\mathcal{O}$ has a distinguished 'multiplication' $\mu \in \mathcal{O}(2)$ and a 'unit' $e \in \mathcal{O}(0)$ such that $\mu \circ_{1} \mu=\mu \circ_{2} \mu \in \mathcal{O}(3)$ and $\mu \circ_{1} e=\mu \circ_{2} e=i d \in \mathcal{O}(1)$.

Let MultSO be the category of multiplicative operads in $\mathcal{A} b$ with unit, i.e. the comma-category $U \mathcal{A} s s / \mathrm{SO}$, where SO is the category of (symmetric) operads. Objects of MultSO are therefore couples $(\mathcal{O}, p)$ of an (ordinary) operad and the structure map. We will, however, drop the structure map from the notation since it will always be clear from the context whether we mean a multiplicative operad with unit or an ordinary operad. Proposition 4.11 of [2] gives an alternative characterization of the category MultSO:

\footnotetext{
${ }^{5}$ Multiplicative nonsymmetric operads were defined in [9].
} 
Proposition 2.3 ([2]). The category of algebras over the $\mathbb{N}$-coloured operad $B$ is isomorphic to the category of multiplicative operads with unit.

Remark 2.4. The operad $\mathcal{F r}=(\mathcal{F} r, \mu, \mathbf{1})$ is clearly the free multiplicative operad with unit generated by $o_{i} \in \mathcal{F} r\left(k_{i}\right), 1 \leq i \leq n$. Proposition 2.1 can also be deduced from Proposition 2.3 and the standard relation between free algebras of a certain type and the operad governing this type of algebras.

We will show that $\check{B}$ is isomorphic to a certain operad of natural operations acting on objects of MultSO.

Definition 2.5. Let $\mathcal{N} a t_{k_{1}, \ldots, k_{n}}^{l}$ be, for $l, k_{1}, \ldots, k_{n} \in \mathbb{N}$, the abelian group of linear maps

$$
\beta_{\mathcal{O}}: \mathcal{O}\left(k_{1}\right) \otimes \cdots \otimes \mathcal{O}\left(k_{n}\right) \rightarrow \mathcal{O}(l)
$$

indexed by multiplicative operads with unit $\mathcal{O} \in$ MultSO such that, for any morphism $\varphi: \mathcal{O} \rightarrow \mathcal{P}$ of multiplicative operads with unit, the diagram

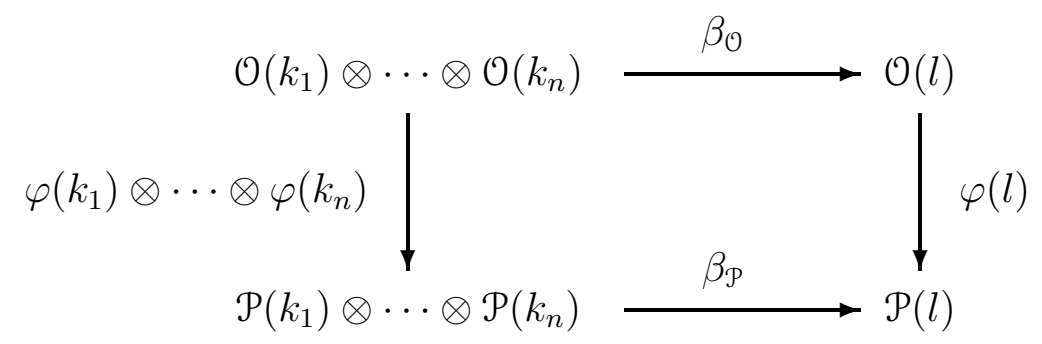

commutes. The abelian groups $\mathcal{N} a t_{k_{1}, \ldots, k_{n}}^{l}$ form an $\mathbb{N}$-coloured operad $\mathcal{N} a t$.

Proposition 2.6. The $\mathbb{N}$-coloured operads $\mathcal{N}$ at and $\check{B}$ are naturally isomorphic.

The proof makes use of a relation between the categories SymCat. and MultSO. Each object $(V, M) \in$ SymCat. has its endomorphism operad

$$
\mathcal{E}_{n d}(V, M):=\left\{\mathcal{E}_{n d_{(V, M)}}(n)\right\}_{n \geq 0} \text { where } \mathcal{E} n d_{(V, M)}(n):=\underline{V}\left(M^{\otimes n}, M\right),
$$

with the convention that $M^{\otimes 0}:=\mathbf{1}$, the unit object of the monoidal category $V$. The unital monoid structure on $M$ clearly determines an operad map $p: U \mathcal{A} s s \rightarrow \mathcal{E}_{n} d_{(V, M)}$ so that $\mathcal{E}_{n d} d_{(V, M)} \in$ MultSO. It is not hard to check that this construction extends to a functor

$$
\mathcal{E} n d: \text { SymCat. } \rightarrow \text { MultSO. }
$$

Observe that $\mathcal{E} n d\left((\Delta S)_{a l g}\right) \cong U \mathcal{A} s s$. With this notation, the operation $\alpha_{(V, M)}$ in (国) is a map

$$
\alpha_{(V, M)}: \mathcal{E} n d_{(V, M)}\left(k_{1}\right) \otimes \cdots \otimes \mathcal{E}_{n d_{(V, M)}}\left(k_{n}\right) \rightarrow \mathcal{E}_{n d_{(V, M)}}(l) .
$$


Lemma 2.7. The functor $\mathcal{E}$ nd has a left adjoint

$$
P: \text { MultSO } \rightarrow \text { SymCat. }
$$

The unit of this adjunction is the identity and the counit

$$
U:\left(P\left(\mathcal{E} n d_{(V, M)}\right), 1\right) \rightarrow(V, M)
$$

is the strict monoidal functor whose effect on the Hom-sets is the identity. Moreover, the functor $\mathcal{E}$ nd is surjective on morphisms (but not full).

Remark 2.8. A functor $F: \mathfrak{C}^{\prime} \rightarrow \mathcal{C}^{\prime \prime}$ is surjective on morphism if for any objects $a^{\prime \prime}, b^{\prime \prime} \in \mathcal{C}^{\prime \prime}$ and a morphism $f^{\prime \prime} \in \mathcal{C}^{\prime \prime}\left(a^{\prime \prime}, b^{\prime \prime}\right)$ there exist objects $a^{\prime}, b^{\prime} \in \mathcal{C}^{\prime}$ and a morphism $f^{\prime} \in \mathcal{C}^{\prime}\left(a^{\prime}, b^{\prime}\right)$ such that $F\left(a^{\prime}\right)=a^{\prime \prime}, F\left(b^{\prime}\right)=b^{\prime \prime}$ and $f^{\prime \prime}=F\left(f^{\prime}\right)$. On the other hand, being full means that, for any objects $a^{\prime}, b^{\prime} \in \mathfrak{C}^{\prime}$, the map $F: \mathfrak{C}^{\prime}\left(a^{\prime}, b^{\prime}\right) \rightarrow \mathfrak{C}^{\prime}\left(F\left(a^{\prime}\right),\left(b^{\prime}\right)\right)$ is an epimorphism. These two conditions are obviously different.

Proof of Lemma 2.7. For an operad $\mathcal{O}$, let $P(\mathcal{O})$ be the PROP freely generated by $\mathcal{O}$, i.e. the additive symmetric monoidal category whose objects are natural numbers with monoidal structure given by the addition, and the spaces of morphisms are

$$
\underline{P(\mathcal{O})}(m, n):=\bigoplus_{i_{1}+\cdots+i_{n}=m} \mathcal{O}\left(i_{1}\right) \otimes \cdots \otimes \mathcal{O}\left(i_{n}\right), \text { for } m, n \geq 0 .
$$

If $\mathcal{O}$ is multiplicative with multiplication $\mu \in \mathcal{O}(2)$ and unit $e \in \mathcal{O}(0), P(\mathcal{O})$ has a distinguished unital monoid $1=(1, \mu, e)$ such that

$$
\mathcal{E} n d_{(P(\mathcal{O}), 1)} \cong \mathcal{O}
$$

in MultSO. It is now straightforward to verify the rest of the lemma.

Proof of Proposition 2.6. We construct mutually inverse morphisms $i: \check{B} \rightarrow \mathcal{N} a t$ and $r: \mathcal{N} a t \rightarrow$ $\check{B}$. For $\alpha \in \check{B}_{k_{1}, \ldots, k_{n}}^{l}$ and $\mathcal{O} \in$ MultSO, let $i(\alpha)_{\mathcal{O}}:=\alpha_{(P(\mathcal{O}), 1)}$. We need to show that the system $i(\alpha)_{\mathcal{O}}$ is natural in the sense that diagram (7) with $i(\alpha)_{\mathcal{O}}=\alpha_{(P(\mathcal{O}), 1)}$ in place of $\beta_{\mathcal{O}}$ and $i(\alpha)_{\mathcal{P}}=\alpha_{(P(\mathcal{P}), 1)}$ in place of $\beta_{\mathcal{P}}$, commutes for each map

$$
\varphi: \mathcal{O} \cong \mathcal{E} n d_{(P(\mathcal{O}), 1)} \rightarrow \mathcal{E} n d_{(P(\mathcal{P}), 1)} \cong \mathcal{P}
$$

of multiplicative operads with unit, with the isomorphisms given by (8). It follows from naturality (3) of $\alpha$ that such diagram commutes if $\varphi$ is induced by a functor $F:(P(\mathcal{O}), 1) \rightarrow(P(\mathcal{P}), 1)$. Lemma 2.7 however shows that all maps $\varphi$ in (9) are induced in this way, so $i(\alpha):=\left\{i(\alpha)_{\mathcal{O}}\right\}$ is a well-defined family in $\mathcal{N} a t_{k_{1}, \ldots, k_{n}}^{l}$. The assignment $\alpha \mapsto i(\alpha)$ obviously extends into an operad map $i: \check{B} \rightarrow \mathcal{N} a t$.

The inverse map $r$ is constructed as follows. For $\beta \in \mathcal{N} a t_{k_{1}, \ldots, k_{n}}^{l}$ and $(V, M) \in$ SymCat., let $^{\prime}$ $r(\beta)_{(V, M)}:=\beta_{\mathcal{E}_{(V, M)}}$. The naturality of the family $r(\beta)_{(V, M)}$ is clear in this case, so $r(\beta)_{(V, M)} \in$ [July 2012] 
$\check{B}_{k_{1}, \ldots, k_{n}}^{l}$. The correspondence $\beta \mapsto r(\beta):=\left\{r(\beta)_{(V, M)}\right\}$ defines a map $r: \mathcal{N} a t \rightarrow \check{B}$ of $\mathbb{N}$-coloured operads.

By definition and (8), one has $i(r(\beta))_{\mathcal{O}}=r(\beta)_{(P(\mathcal{O}), 1)}=\beta_{\mathcal{E}_{(P(\mathcal{O}), 1)}}=\beta_{\mathcal{O}}$, so $i r=i d_{\mathfrak{N a t}}$. For the opposite composition, one gets $r(i(\alpha))_{(V, M)}=i(\alpha)_{\mathcal{E} n d_{(V, M)}}=\alpha_{\left(P\left(\mathcal{E n d}_{(V, M)}\right), 1\right)}$. To show that $r i=i d_{\check{B}}$, i.e. that

$$
\alpha_{\left(P\left(\mathcal{E n d}_{(V, M)}\right), 1\right)}=\alpha_{(V, M)} \text { for each }(V, M) \in \text { SymCat. }_{\bullet}
$$

one uses naturality with respect to the counit $U:\left(P\left(\mathcal{E} n d_{(V, M)}\right), 1\right) \rightarrow(V, M)$. By Lemma 2.7 again the following diagram commutes

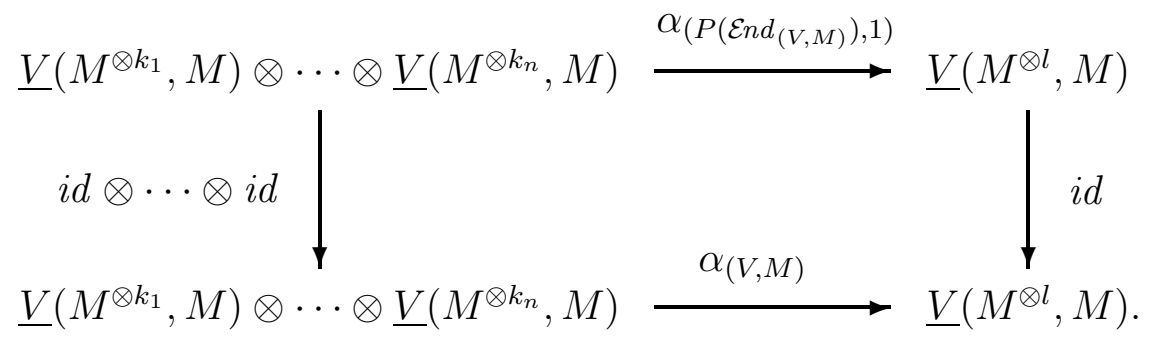

This proves $(10)$ and establishes $r i=i d_{\check{B}}$ as well.

The last step in the proof of Theorem A is:

Proposition 2.9. The $\mathbb{N}$-coloured operads $\mathcal{N}$ at and $B$ are naturally isomorphic.

Proof. Denote, as in (6), by $\mathcal{F r}$ be the free multiplicative operad with unit generated by $n$ operations $o_{i} \in \mathcal{F} r\left(k_{i}\right)$ of arities $k_{i}, 1 \leq i \leq n$. Let $\mathcal{O}$ be an arbitrary multiplicative operad with unit. Given elements $x_{i} \in \mathcal{O}\left(k_{i}\right), 1 \leq i \leq n$, there exists a unique map $F_{x_{1}, \ldots, x_{n}}^{\mathcal{O}}: \mathcal{F} r \rightarrow \mathcal{O}$ of multiplicative operads with unit specified by $F_{x_{1}, \ldots, x_{n}}^{\mathcal{O}}\left(o_{i}\right):=x_{i}, 1 \leq i \leq n$. Observe that

$$
F_{o_{1}, \ldots, o_{n}}^{\mathcal{F} r}=i d_{\mathcal{F} r}
$$

and that, for each map $\varphi: \mathcal{O} \rightarrow \mathcal{P}$ of multiplicative operads with unit, one has

$$
F_{x_{1}, \ldots, x_{n}}^{\mathcal{O}}=\varphi \circ F_{\varphi\left(x_{1}\right), \ldots, \varphi\left(x_{n}\right)}^{\mathcal{P}} .
$$

For a natural operation $\beta \in \mathcal{N} a t_{k_{1}, \ldots, k_{n}}^{l}$ and $x_{i} \in \mathcal{O}\left(k_{i}\right) 1 \leq i \leq n$ as above, one has, by naturality (17), the commutative diagram

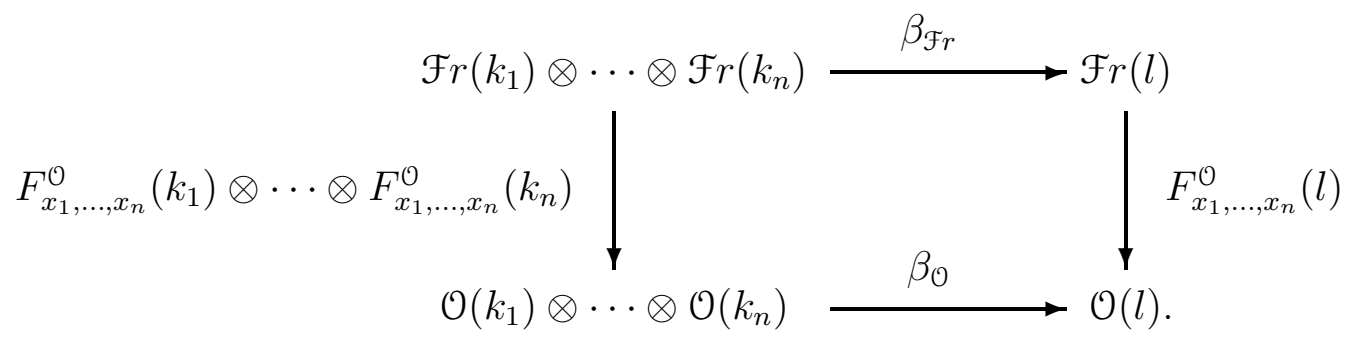

[July 2012] 
We will need also a particular case of (13) when $\mathcal{O}=\mathcal{F} r$ and $x_{i}=u_{i} o_{i}$, for some scalars $u_{i} \in \mathbb{Z}$, $1 \leq i \leq n$ :

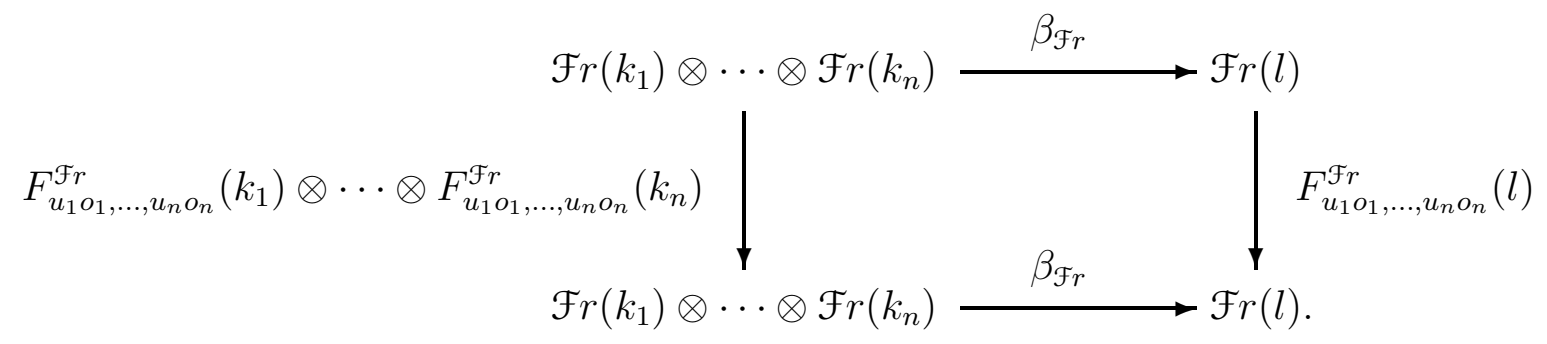

Recall that $\mathcal{F} r_{1, \ldots, 1}(l)$ denotes the subspace of elements of $\mathcal{F} r(l)$ containing each generator $o_{1}, \ldots, o_{n}$ precisely once. We begin the proof by observing that each natural operation $\beta \in$ $\mathcal{N} a t_{k_{1}, \ldots, k_{n}}^{l}$ determines an element $\xi(\beta) \in \mathcal{F} r(l)$ by

$$
\xi(\beta):=\beta_{\mathcal{F r}}\left(o_{1} \otimes \cdots \otimes o_{n}\right) \in \mathcal{F} r(l) .
$$

We need to show that $\xi(\beta)$ in fact belongs to $\mathcal{F} r_{1, \ldots, 1}(l) \cong B_{k_{1}, \ldots, k_{n}}^{l}$. Clearly, $\mathcal{F} r(l)$ decomposes as

$$
\mathcal{F} r(l)=\bigoplus_{j_{1}, \ldots, j_{n} \geq 0} \mathcal{F} r_{j_{1}, \ldots, j_{n}}(l),
$$

where $\mathcal{F} r_{j_{1}, \ldots, j_{n}}(l) \subset \mathcal{F} r(l)$ is the subspace of elements having precisely $j_{i}$ instances of $o_{i}$ for each $1 \leq i \leq n$. The endomorphism $F_{u_{1} o_{1}, \ldots, u_{n} o_{n}}^{\mathcal{F r}}: \mathcal{F r} \rightarrow \mathcal{F r}$ acts on $\mathcal{F} r_{j_{1}, \ldots, j_{n}}(l)$ by the multiplication with $u_{1}^{j_{1}} \cdots u_{n}^{j_{n}}-\mathcal{F} r_{j_{1}, \ldots, j_{n}}(l) \subset \mathcal{F} r(l)$ is, in fact, characterized by this property. The element $\xi(\beta)$ uniquely decomposes as $\xi(\beta)=\sum_{j_{1}, \ldots, j_{n} \geq 0} \xi(\beta)_{j_{1}, \ldots, j_{n}}$, for some $\xi(\beta)_{j_{1}, \ldots, j_{n}} \in \mathcal{F} r_{j_{1}, \ldots, j_{n}}(m)$.

Let us turn our attention to diagram ([4]). By the definition of the map $\mathcal{F}_{u_{1} o_{1}, \ldots, u_{n} o_{n}}^{\mathcal{F} r}$, one has

$$
\beta_{\mathcal{F} r}\left(F_{u_{1} o_{1}, \ldots, u_{n} o_{n}}^{\mathcal{F} r}\left(k_{1}\right) \otimes \cdots \otimes F_{u_{1} o_{1}, \ldots, u_{n} o_{n}}^{\mathcal{F} r}\left(k_{n}\right)\right)\left(o_{1} \otimes \cdots \otimes o_{n}\right)=\beta_{\mathcal{F} r}\left(u_{1} O_{1} \otimes \cdots \otimes u_{n} o_{n}\right),
$$

while the linearity of $\beta_{\mathcal{F r}}$ implies

$$
\beta_{\mathcal{F r}}\left(u_{1} O_{1} \otimes \cdots \otimes u_{n} O_{n}\right)=u_{1} \cdots u_{n} \cdot \beta_{\mathcal{F r}}\left(o_{1} \otimes \cdots \otimes o_{n}\right)=u_{1} \cdots u_{n} \cdot \xi(\beta) .
$$

On the other hand

$$
F_{u_{1} o_{1}, \ldots, u_{n} o_{n}}^{\mathcal{F} r}(l)\left(\beta_{\mathcal{F r} r}\right)\left(o_{1} \otimes \cdots \otimes o_{n}\right)=F_{u_{1} o_{1}, \ldots, u_{n} o_{n}}^{\mathcal{F} r}(l)(\xi(\beta))=\sum_{j_{1}, \ldots, j_{n} \geq 0} u_{1}^{j_{1}} \cdots u_{n}^{j_{n}} \cdot \xi(\beta)_{j_{1}, \ldots, j_{n}},
$$

therefore the commutativity of (14) means that

$$
u_{1} \cdots u_{n} \cdot \xi(\beta)=\sum_{j_{1}, \ldots, j_{n} \geq 0} u_{1}^{j_{1}} \cdots u_{n}^{j_{n}} \cdot \xi(\beta)_{j_{1}, \ldots, j_{n}}
$$

for each $u_{1}, \ldots, u_{n} \in \mathbb{Z}$. We conclude that $\xi(\beta)_{j_{1}, \ldots, j_{n}}=0$ if $\left(j_{1}, \ldots, j_{n}\right) \neq(1, \ldots, 1)$, so $\xi(\beta)=$ $\xi(\beta)_{1, \ldots, 1} \in \mathcal{F} r_{1, \ldots, 1}(l)$.

Let us prove that, vice versa, each element $\eta \in \mathcal{F} r_{1, \ldots, 1}(l) \cong B_{k_{1}, \ldots, k_{n}}^{l}$ determines a natural operation $\gamma(\eta) \in \mathcal{N} a t_{k_{1}, \ldots, k_{n}}^{l}$ by the formula

$$
\gamma(\eta)_{\mathcal{O}}\left(x_{1} \otimes \cdots \otimes x_{n}\right)=F_{x_{1}, \ldots, x_{n}}^{\mathcal{O}}(l)(\eta), \text { for each } x_{i} \in \mathcal{O}\left(k_{i}\right), 1 \leq i \leq n .
$$


The linearity of the map $\gamma(\eta)_{\mathcal{O}}: \mathcal{O}\left(k_{1}\right) \otimes \cdots \mathcal{O}\left(k_{n}\right) \rightarrow \mathcal{O}(l)$ defined in this way easily follows from the fact that $\eta$ belongs to the subspace $\mathcal{F} r_{1, \ldots, 1}(l) \subset \mathcal{F} r(l)$. The commutativity of diagram (đ) for the family $\gamma(\eta)=\{\gamma(\eta)\}_{\mathcal{O}}$ defined by (16) is equivalent to

$$
\varphi\left(\gamma(\eta)_{\mathcal{O}}\right)\left(x_{1} \otimes \cdots \otimes x_{n}\right)=\gamma(\eta)_{\mathcal{P}}\left(\varphi\left(k_{1}\right) \otimes \cdots \otimes \varphi\left(k_{n}\right)\right)\left(x_{1} \otimes \cdots \otimes x_{n}\right)
$$

for each $x_{i} \in \mathcal{O}\left(k_{i}\right), 1 \leq i \leq n$. This, by the definition (16) of $\gamma(\eta)$, means

$$
F_{x_{1}, \ldots, x_{n}}^{\mathcal{O}}(l)(\eta)=\varphi \circ F_{\varphi\left(x_{1}\right), \ldots, \varphi\left(x_{n}\right)}^{\mathcal{P}}(l)(\eta)
$$

which follows from (12). So $\gamma(\eta)=\left\{\gamma(\eta)_{\mathcal{O}}\right\}$ is a well-defined natural operation in $\mathcal{N} a t_{k_{1}, \ldots, k_{n}}^{l}$.

Let us prove that the maps $\xi: \mathcal{N} a t_{k_{1}, \ldots, k_{n}}^{l} \rightarrow \mathcal{F} r_{1, \ldots, 1}(l)$ and $\gamma: \mathcal{F} r_{1, \ldots, 1}(l) \rightarrow \mathcal{N} a t_{k_{1}, \ldots, k_{n}}^{l}$ defined by the above correspondences are mutual inverses. We have, for $\eta \in \mathcal{F} r_{1, \ldots, 1}(l)$,

$$
\xi(\gamma(\eta))=\gamma(\eta)_{\mathcal{F r}}\left(o_{1} \otimes \cdots \otimes o_{n}\right)=F_{o_{1}, \ldots, o_{n}}^{\mathcal{F r}}(l)(\eta)=\eta,
$$

where the last equality follows from (11). For the opposite composition, one has

$$
\begin{aligned}
\gamma(\xi(\beta))_{\mathcal{O}}\left(x_{1} \otimes \cdots \otimes x_{n}\right) & =F_{x_{1}, \ldots, x_{n}}^{\mathcal{O}}(l)(\xi(\beta))=F_{x_{1}, \ldots, x_{n}}^{\mathcal{O}}(l)\left(\beta_{\mathcal{F r}}\right)\left(o_{1} \otimes \cdots o_{n}\right) \\
& =\beta_{\mathcal{O}}\left(F_{x_{1}, \ldots, x_{n}}^{\mathcal{O}}\left(k_{1}\right)\left(o_{1}\right) \otimes \cdots \otimes F_{x_{1}, \ldots, x_{n}}^{\mathcal{O}}\left(k_{n}\right)\left(o_{n}\right)\right) \\
& =\beta_{\mathcal{O}}\left(x_{1} \otimes \cdots \otimes x_{n}\right),
\end{aligned}
$$

where we used the commutativity of (13) and the definition of $F_{x_{1}, \ldots, x_{n}}^{\mathcal{O}}$. The proof is finished by observing that the two mutually inverse families of maps $\xi: \mathcal{N} a t_{k_{1}, \ldots, k_{n}}^{l} \rightarrow \mathcal{F} r_{1, \ldots, 1}(l) \cong B_{k_{1}, \ldots, k_{n}}^{l}$ and $\gamma: B_{k_{1}, \ldots, k_{n}}^{l} \cong \mathcal{F} r_{1, \ldots, 1}(l) \rightarrow \mathcal{N} a t_{k_{1}, \ldots, k_{n}}^{l}$ define two mutually inverse homomorphisms $\xi: \mathcal{N} a t \rightarrow$ $B$ and $\gamma: B \rightarrow \mathcal{N}$ at of $\mathbb{N}$-coloured operads.

We have a nonsymmetric variant of Proposition 2.3.

Proposition 2.10. The category of algebras over the $\mathbb{N}$-coloured operad $T$ is isomorphic to the category of multiplicative nonsymmetric operads with unit.

\section{Crossed interval groups}

In this section we introduce crossed interval groups. We believe that this new concept is important on its own and we therefore decided to include also some results and examples not directly related to our immediate applications. The reader who is not interested in this more general picture may read only the material necessary for this paper - Definition 3.2, Example 3.4, Proposition 3.9, Definition 3.10, Lemma 3.11, Lemma 3.12 - and skip the rest.

The category of intervals $\mathcal{J}$ is the category whose objects are the linearly ordered sets $\langle n\rangle:=$ $\{-1,0, \ldots, n, n+1\}, n \geq-1$, and morphisms non-decreasing maps $f:\langle m\rangle \rightarrow\langle n\rangle$ such that $f(-1)=-1$ and $f(m+1)=n+1$. The points $-1, n+1$ are called the boundary points of $\langle n\rangle$ and the ordinal $[n]:=\{0, \ldots, n\}$ is the interior of $\langle n\rangle$. Intuitively, one may think of 
$\langle n\rangle$ as of the interval with endpoints -1 and $n+1$; morphisms in $\mathcal{J}$ are then non-decreasing, boundary preserving maps of intervals. The following statement goes back, probably, to Gabriel and Zisman [8, Section III.1.1] but became known under the name Joyal's duality due to an influential preprint [11]. The reader who is interested to understand the details of Joyal's duality including higher dimensional generalization is referred to 16].

Proposition 3.1 (Joyal's duality). The category $\mathcal{J}$ is isomorphic to the opposite category $\Delta^{\mathrm{op}}$ of the (skeletal) category $\Delta$ of nonempty finite ordered sets and their non-decreasing maps.

Proof. To construct the isomorphism joy : $\Delta^{\mathrm{op}} \cong \mathcal{J}$, recall that $\Delta$ has objects the finite ordinals $[n]=\{0, \ldots, n\}, n \geq 0$. Morphism of $\Delta$ are generated by the face maps $\delta_{i}:[n-1] \rightarrow[n]($ misses $i$ ) and the degeneracy maps $\sigma_{i}:[n+1] \rightarrow[n]$ (hits $i$ twice), $i=0, \ldots, n$. Then $j o y([n]):=\langle n-1\rangle$, for $n \geq 0$. Moreover, $d_{i}=\operatorname{joy}\left(\delta_{i}\right):\langle n-1\rangle \rightarrow\langle n-2\rangle$ is the map that hits $i-1$ twice, and $s_{i}=j o y\left(\sigma_{i}\right):\langle n-1\rangle \rightarrow\langle n\rangle$ the map that misses $i$.

The category $\mathcal{J}$ contains a subcategory of open maps. The objects of this subcategory are intervals and the morphisms are interval morphisms $f:\langle m\rangle \rightarrow\langle n\rangle$ which preserve the interiors i.e. $f(\{0, \ldots, m\}) \subset\{0, \ldots, n\}$. This subcategory is isomorphic to the (skeletal) category of all finite ordinals called $\Delta_{\text {alg }}$ (algebraic $\Delta$ ). The category $\Delta_{\text {alg }}$ has a well-known universal property - it is a monoidal category freely generated by a monoid. The following definition is motivated by [0, Definition 1.1].

Definition 3.2. A crossed interval group $\mathcal{H}$ is a sequence of groups $\left\{H^{n}\right\}_{n \geq 0}$ equipped with the following structure:

(a) a small category $\mathcal{J} H$ called the associated category such that the objects of $\mathcal{J} H$ are the ordinals $\langle n\rangle=\{-1,0, \ldots, n, n+1\}, n \geq-1$,

(b) $\mathcal{J} H$ contains $\mathcal{J}$ as a subcategory,

(c) $\operatorname{Aut}_{\mathcal{J}}(\langle n\rangle)=\left(H^{n+1}\right)^{\mathrm{op}}, n \geq-1$, and

(d) any morphism $f:\langle m\rangle \rightarrow\langle n\rangle$ in $\mathcal{J} H$ can be uniquely written as $\phi \circ h$, where $\phi \in$ $\operatorname{Hom}_{\mathcal{J}}(\langle m\rangle,\langle n\rangle)$ and $h \in\left(H^{m+1}\right)^{\text {op }}$.

We would like to extend the Joyal's duality to the case of crossed interval groups. To this end, we need

Definition 3.3. A crossed cosimplicial group $\mathcal{H}$ is a sequence of groups $\left\{H^{n}\right\}_{n \geq 0}$ equipped with the following structure:

(a) a small category $H \Delta$ such that the objects of $H \Delta$ are the ordinals $[n]=\{0,, \ldots, n\}$, $n \geq 0$,

(b) $H \Delta$ contains $\Delta$ as a subcategory, 
(c) $\operatorname{Aut}_{H \Delta}([n])=H^{n}, n \geq 0$, and

(d) any morphism $f:[m] \rightarrow[n]$ in $H \Delta$ can be uniquely written as $h \circ \phi$, where $\phi \in$ $\operatorname{Hom}_{\Delta}([m],[n])$ and $h \in H^{n}$.

Now, given a crossed interval group $\mathcal{H}$ we construct its Joyal's dual crossed cosimplicial group $J \mathcal{H}$ with the associated category $(J H) \Delta$ by taking the same sequence of groups $\left\{H^{n}\right\}_{n \geq 0}$ and putting $(J H) \Delta([n],[m]):=\mathcal{J} H(\langle m-1\rangle,\langle n-1\rangle)$. One can easily check using the ordinary Joyal's duality that we thus obtain a crossed cosimplicial group. Moreover, we also obtain an isomorphism of the associated categories $j_{H}:((J H) \Delta)^{\mathrm{op}} \rightarrow \mathfrak{J} H$. We therefore see that crossed interval groups and crossed cosimplicial groups are equivalent notions.

An important crossed interval group is introduced in the following example.

Example 3.4. Let $J S$ be the category whose objects are the ordinals $\langle n\rangle=\{-1,0, \ldots, n, n+1\}$, $n \geq-1$, and morphisms are maps $f:\langle m\rangle \rightarrow\langle n\rangle$ such that

(a) a linear order on each non-empty fiber $f^{-1}(i), i \in\langle n\rangle$ is specified,

(b) $f$ preserves the endpoints and their order, that is $f(-1)=-1$ and $f(m+1)=n+1$, and

(c) -1 is the minimal element in $f^{-1}(-1)$ and $m+1$ is the maximal element in $f^{-1}(n+1)$.

The group $\operatorname{Aut}_{\mathcal{T}}(\langle n\rangle)$ equals the symmetric group $S_{n+1}$, whence the notation. We leave as an exercise to verify that the $\mathfrak{J} S$ defined above is an associated category of a crossed interval group. We denote this crossed interval group $\mathcal{S}$.

Let $\Delta S$ be the category whose objects are the ordinals $[n]:=\{0, \ldots, n\}$ and morphisms are maps $g:[m] \rightarrow[n]$ with a specified linear order on the fibers $g^{-1}(i), i \in[n]$. Recall that $\Delta S$, called the symmetric category, is an associated category of a crossed simplicial group $S_{*}$ in the sense of [7, Definition 1.1]. There is also an 'algebraic' version of the symmetric category $(\Delta S)_{\text {alg }}$ introduced by Day and Street in $\mathbb{A}$, which has a universal property similar to $\Delta_{a l g}$ : the category $(\Delta S)_{a l g}$ is the symmetric monoidal category freely generated by a (noncommutative) monoid.

Analogously to the inclusion $\Delta_{a l g} \hookrightarrow \mathcal{J}$, one has the inclusion

$$
I:(\Delta S)_{\text {alg }} \hookrightarrow \mathcal{J} S
$$

such that $I([n]):=\langle n\rangle$. If $g:[m] \rightarrow[n]$ is a morphism in $(\Delta S)_{\text {alg }}$, then $I(g)$ is the unique morphism $f:\langle m\rangle \rightarrow\langle n\rangle$ such that $\left.f\right|_{\{0, \ldots, m\}}=g$. The image of $I$ is the subcategory of open maps, i.e. maps $f:\langle m\rangle \rightarrow\langle n\rangle$ such that $f(\{0, \ldots, m\}) \subset\{0, \ldots, n\}$. Likewise, there is the inclusion

$$
\iota: \mathcal{J} S \hookrightarrow \Delta S
$$

such that $\iota(\langle n\rangle):=[n+2]$ and, for $f:\langle m\rangle \rightarrow\langle n\rangle, g=\iota(f)$ is the map with $g(i):=f(i-1)$, for $i \in[n+2]$. 
Example 3.5. Another, rather trivial example of a crossed interval group is the 'constant' group $z_{2}$. The objects are the intervals $\langle n\rangle, n \geq-1$, and the morphisms are maps $f:\langle m\rangle \rightarrow\langle n\rangle$ such that $f$ restricted to the interior of the interval is order preserving and $f$ preserves the boundary, that is, $f(\{-1, m+1\})=\{-1, n+1\}$. It is not hard to see that all the automorphisms groups in this category are $\mathbb{Z}_{2}$.

Example 3.6. The crossed interval group Flip. The objects are again the intervals. The morphisms from $\langle n\rangle$ to $\langle m\rangle$ are maps $f:\langle m\rangle \rightarrow\langle n\rangle$ that preserve the boundary, which are either order preserving or order reversing. Again, it is not difficult to see that all automorphism groups are $\mathbb{Z}_{2}$. The unique nontrivial automorphism of an interval is the flip around its center. The crossed interval groups $\mathcal{Z}_{2}$ and $\mathcal{F l i p}$ are, however, not isomorphic.

Example 3.7. One can construct an example of a crossed interval group $\mathcal{B}$ raid if one replaces symmetric groups by braid groups in Example 3.4. The braid analogue of Example 3.5 will be the 'constant' crossed interval group 2 . We do not know if there is a braid analogue of the crossed interval group $\mathcal{F}$ lip of Example 3.6.

Remark 3.8. The examples of $z_{2}$ and $\mathcal{F l i p}$ indicate that the classification of the crossed interval groups is, probably, more subtle than the classification of crossed simplicial groups given in [7].

One advantage of the crossed interval groups over crossed cosimplicial groups is the same variance of morphisms as in Loday-Fiedorowicz crossed simplicial groups. So, many basic results for crossed simplicial groups can be carried over to crossed interval groups without changing the formulas from [7]. For example, we have that for any $h \in H^{n}$ and any $\phi \in \mathcal{J}(\langle m\rangle,\langle n\rangle)$ there exist unique $\phi^{*}(h) \in H^{m}$ and $h^{*}(\phi) \in \mathcal{J}(\langle m\rangle,\langle n\rangle)$ such that $h \circ \phi=h^{*}(\phi) \circ \phi^{*}(h)$. Using these correspondences one can give an alternative characterization of crossed interval groups repeating [7], Proposition 1.6] verbatim.

Let us define an automorphism of an interval $\langle n\rangle$ as a bijection $\langle n\rangle$ to $\langle n\rangle$ which preserves the set of boundary points. Clearly, the group Aut $\langle n\rangle$ of all automorphisms is isomorphic to $S_{n+1} \times \mathbb{Z}_{2}$, where $S_{n+1}$ denotes the symmetric group on $n+1$ elements and $\mathbb{Z}_{2}:=\mathbb{Z} / 2 \mathbb{Z}$.

The following characterization of the crossed interval groups is slightly more complicated than its crossed simplicial analogue (see Proposition 1.7 from [7]).

Proposition 3.9. A crossed interval group $\mathcal{H}$ is a cosimplicial set $H^{\bullet}=\left\{H^{n}\right\}_{n \geq 0}$ such that each $H^{n}$ is a group, together with a group homomorphism

$$
\rho_{n}: H^{n} \rightarrow \operatorname{Aut}\langle n-1\rangle \cong S_{n} \times \mathbb{Z}_{2}
$$

for each $n \geq 0$, such that, for $h, h^{\prime} \in H^{n}$,

(1) $d_{i}\left(h h^{\prime}\right)=d_{\bar{h}(i)}\left(h^{\prime}\right) d_{i}(h)$, where $\bar{h}(i):=\rho_{n}(h)(i-1)+1,0 \leq i \leq n+1$, 
(2) $s_{i}\left(h h^{\prime}\right)=s_{\underline{h}(i)}\left(h^{\prime}\right) s_{i}(h)$, where $\underline{h}(i):=\rho_{n}(h)(i), 0 \leq i \leq n-1$.

(3) Moreover, the following set diagrams are commutative:

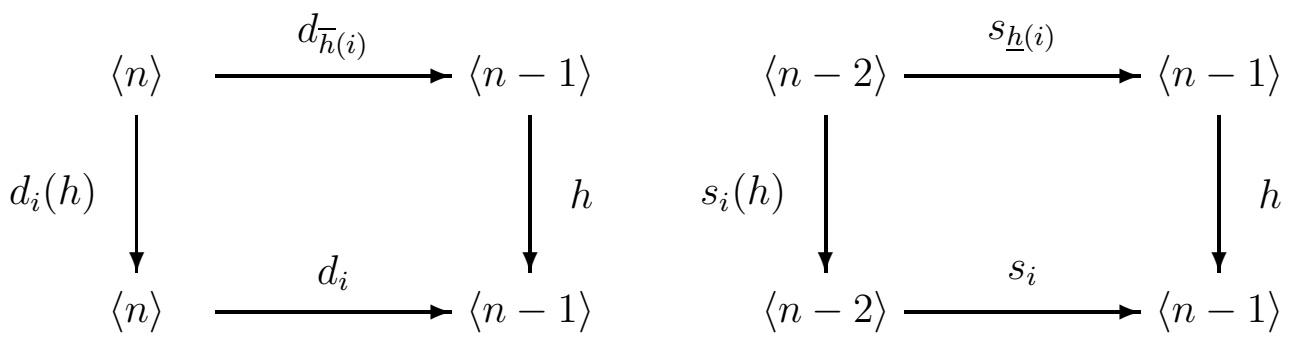

where $0 \leq i \leq n+1$ in the first and $0 \leq i \leq n-1$ in the second diagram.

The proof is basically identical to the proof of Proposition 1.7 in [7] but we have to take into account that among the coface operators $d_{i}$ in $\mathcal{J}$ the first and the last operators are not open maps of intervals. One can check that under the correspondence $h^{*}: \mathcal{J}(\langle m\rangle,\langle n\rangle) \rightarrow \mathcal{J}(\langle m\rangle,\langle n\rangle)$ the open maps go to the open maps, so the first and the last operators can only be stable or permuted by $h^{*}$. This explains the factor $\mathbb{Z}_{2}$ in (18).

We also observe that $\rho_{*}$ is a homomorphism of crossed interval groups $\mathcal{H} \rightarrow \mathcal{S} \times \mathcal{Z}_{2}$, so the value of $\rho_{n}$ is determined by its action on the set of open coface operators $\left\{d_{i} ; 1 \leq i \leq n\right\}$ or equally on the set of codegenaracies (which are automatically open maps of intervals) $\left\{s_{i} ; 0 \leq i \leq n-1\right\}$, and the homomorphism $\rho_{0}: H^{0} \rightarrow \mathbb{Z}_{2}$.

Definition 3.10. Let $\mathcal{H}$ be a crossed interval group and let $\mathcal{C}$ be a category. An $\mathcal{H}$-object in $\mathcal{C}$ is a functor $X:(\mathcal{J} H)^{\text {op }} \rightarrow \mathcal{C}$. A morphism between two $\mathcal{H}$-objects is a natural transformation of functors.

Similarly to the crossed simplicial case we have the following simple characterization of $\mathcal{H}$ objects (compare with Lemma 4.2 of [7]). We formulate it, as well as Lemma 3.12 below, in the more intuitive language of elements, though, of course, objects of a general cocomplete category do not have 'elements.' The concerned reader may easily translate the statements into the formally correct language of arrows.

Lemma 3.11. The category of $\mathcal{H}$-objects in a cocomplete category $\mathcal{C}$ is equivalent to the category whose objects are cosimplicial objects $X^{\bullet}$ in $\mathcal{C}$ equipped with the following additional structure:

(1) right group actions $X^{n} \times H^{n} \rightarrow X^{n}, n \geq 0$,

(2) coface relations $d_{i}(x h)=d_{\bar{h}(i)}(x) d_{i}(h)$, for $x \in X^{n}, h \in H^{n}, 0 \leq i \leq n+1$,

(3) codegeneracy relations $s_{i}(x h)=s_{\underline{h}(i)}(x) s_{i}(h)$, for $x \in X^{n}, h \in H^{n}, 0 \leq i \leq n-1$,

and whose morphisms are cosimplicial morphisms which are degreewise equivariant. 
In the above lemma, $\bar{h}(i)$ and $\underline{h}(i)$ have the same meaning as in Proposition 3.9. There is a natural restriction functor $\mathcal{U}_{\mathcal{H}}$ from the category of $\mathcal{H}$-objects in $\mathcal{C}$ to the category of cosimplicial objects in $\mathcal{C}$. This functor has a left (right) adjoint provided $\mathcal{C}$ is cocomplete (complete). We will denote the left adjoint by $\mathcal{F}_{\mathcal{H}}$. It is easy to get explicit formulas for $\mathcal{F}_{\mathcal{H}}$ similar to the formulas from [7], Definition 4.3]:

Lemma 3.12. Let $X=X^{\bullet}$ be a cosimplicial object in a cocomplete category $\mathcal{C}$. Then $\mathcal{F}_{\mathcal{H}}(X)$ is an $\mathcal{H}$-object with $\mathcal{F}_{\mathcal{H}}(X)^{n}:=X^{n} \times H^{n}, n \geq 0$, and with cofaces and codegeneracies given by

$$
\begin{gathered}
d_{i}(x, h):=\left(d_{\bar{h}(i)}(x), d_{i}(h)\right), \text { for } x \in X^{n}, h \in H^{n}, 0 \leq i \leq n+1, \\
s_{i}(x, h):=\left(s_{\underline{h}(i)}(x), s_{i}(h)\right), \text { for } x \in X^{n}, h \in H^{n}, 0 \leq i \leq n-1 .
\end{gathered}
$$

The unit $\iota: X \mapsto F_{\mathcal{H}}(X)$ of the monad $F_{\mathcal{H}}$ generated by the adjunction $\mathcal{F}_{\mathcal{H}} \dashv \mathcal{U}_{\mathcal{H}}$ is given by the cosimplicial map $\iota(x):=(x, 1)$.

\section{A homotopy equivalence of Cosimplicial totalizations.}

This section is devoted to a result about cosimplicial totalizations of $\mathcal{S}$-chain complexes, where $\mathcal{S}$ is the crossed interval group introduced in Example 3.4. We recall first the notion of cosimplicial totalization that associates to a cosimplicial chain complex $C_{*}^{\bullet}$ a chain complex $\overline{\operatorname{Tot}}\left(C_{*}^{\bullet}\right)$. The main result of this section, Theorem 4.4, states that the cochain complex associated to $F_{\mathcal{S}}\left(C_{*}^{\bullet}\right)$, where $C_{*}^{\bullet}$ is a cosimplicial chain complex with finitely generated torsion-free components, is canonically homotopy equivalent to the cochain complex associated to $C_{*}^{\bullet}$. Our main technical tool will be a cochain version of the method of acyclic models developed in Appendix $\mathrm{A}$.

This result should be considered as dual to the result about crossed simplicial group $\Delta S$ which says that the geometric realization of simplicial topological space $F_{\Delta S}\left(X_{\bullet}\right)$ is homotopy equivalent to the geometric realization of $X_{\bullet}$. The proof of this statement is relatively easy. Theorem 5.3 from [7] provides an equivariant homeomorphism of geometric realizations

$$
\left|F_{G}\left(X_{\bullet}\right)\right| \rightarrow\left|G_{\bullet}\right| \times\left|X_{\bullet}\right|
$$

for any crossed simplicial group $G$. Then it is enough to observe that the geometric realization of the simplicial set $\Delta S_{\bullet}$ is contractible by explicitly constructing a simplicial contraction.

Unfortunately, the methods of [0] do not work for the crossed interval groups. So we need to develop a different approach. Our argument is based on a corollary (Proposition A.5) to a version of Acyclic Model Theorem for cochain functors which we formulate in the Appendix $\mathrm{A}$.

Let Chain be the category of $\mathbb{Z}$-graded chain complexes of abelian groups. It is a closed symmetric monoidal category with respect to the usual tensor product of chain complexes. Let Chain $^{\Delta}$ be the category of cosimplicial objects in Chain. Objects of Chain $^{\Delta}$ are systems $C_{*}^{\bullet}=$ [July 2012] 
$\left(C_{*}^{n}, \partial\right), n \geq 0$, of chain complexes with face and degeneracy operators $d_{i}: C_{*}^{n-1} \rightarrow C_{*}^{n}, s_{i}:$ $C_{*}^{n+1} \rightarrow C_{*}^{n}, 0 \leq i \leq n$, which are chain maps and satisfy the standard cosimplicial identities.

Let CochChain be the category of non-negatively graded cochain complexes in the category Chain. Objects of CochChain are systems $\left(E_{*}^{*}, d, \partial\right)$ consisting of abelian groups $E_{k}^{m}, m \geq 0$, $k \in \mathbb{Z}$, and linear maps $d: E_{k}^{m} \rightarrow E_{k}^{m+1}$ (horizontal differentials), $\partial: E_{k+1}^{m} \rightarrow E_{k}^{m}$ (vertical differentials) that square to zero and satisfy $\partial d+d \partial=0$.

Each cosimplicial chain complex $C_{*}^{\bullet} \in$ Chain $^{\Delta}$ determines an object $C_{*}^{*}$ of Coch hain, with the differential $d: C_{k}^{m} \rightarrow C_{k}^{m+1}$ given by the standard formula

$$
d(c):=\sum_{0 \leq i \leq m+1}(-1)^{i+m} d_{i}(c), c \in C_{k}^{m}, m \geq 0, k \in \mathbb{Z} .
$$

The correspondence $C_{*}^{\bullet} \mapsto C_{*}^{*}$ defines the functor 'replace bullet by star' $\mathcal{N}:$ Chain ${ }^{\Delta} \rightarrow$ eochehain.

For $E_{*}^{*} \in$ CochChain denote by $\left|E_{*}^{*}\right|^{*}$ the cochain complex with $\left|E_{*}^{*}\right|^{m}:=\prod_{n-k=m} E_{k}^{n}$, with the differential given by

$$
d\left(a^{0}, a^{1}, a^{2}, \ldots\right):=\left(-\partial a^{0}, d a^{0}-\partial a^{1}, d a^{1}-\partial a^{2}, \ldots\right)
$$

for $\left(a^{0}, a^{1}, a^{2}, \ldots\right) \in E_{*}^{0} \times E_{*}^{1} \times E_{*}^{2} \times \cdots$. Finally, define the total complex of a cosimplicial chain complex $C_{*}^{\bullet}$ to be the cochain complex $\overline{\operatorname{Tot}}\left(C_{*}^{\bullet}\right)^{*}:=\left|\mathcal{N}\left(C_{*}^{\bullet}\right)\right|^{*}$. The bar over Tot indicates that we totalize with respect to the cosimplicial structure. So we have three functors forming a commutative triangle:

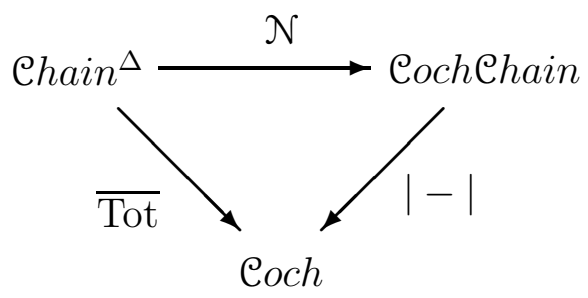

Warning. Objects of CochChain are particular types of bicomplexes of abelian groups (with a slightly unusual degree convention), but our definition of $\left|E_{*}^{*}\right|^{*}$ differs from the traditional definition of the total complex of a bicomplex in that it involves the direct product instead of the direct sum.

Example 4.1. The components of the operad $\mathcal{B}$ form a cosimplicial, non-negatively graded chain complex $B_{*}^{\bullet}(n)$ with $B_{m}^{q}(n)=\bigoplus_{k_{1}+\cdots+k_{n}=m} B_{k_{1}, \ldots, k_{n}}^{q}$. The corresponding total space $\overline{\operatorname{Tot}}\left(B_{*}^{\bullet}(n)\right)$ equals the arity $n$ component $\mathcal{B}(n)$ of the $\mathrm{dg}$ operad $\mathcal{B}$. Another example is the cosimplicial chain complex determined, in the same manner, by the components of the suboperad $\mathcal{T} \subset \mathcal{B}$. 
To simplify the notation, we will drop, in the rest of this section, the stars $*$ from the notation whenever the nature of the corresponding object is clear from the context. The unit of the monad from Lemma 3.12 induces the canonical inclusion of cosimplicial chain complexes

$$
\iota: C^{\bullet} \rightarrow F_{\mathcal{S}}\left(C^{\bullet}\right)
$$

given by $\iota(a):=(a, 1)$.

Definition 4.2. The miraculous map $m: \mathcal{N}\left(F_{\mathcal{S}}\left(C^{\bullet}\right)\right) \rightarrow \mathcal{N}\left(C^{\bullet}\right)$ is defined by the formula

$$
m(a, \sigma):=\operatorname{sgn}(\sigma) a, a \in C^{q}, \sigma \in S_{q}, q \geq 0 .
$$

We call $m$ miraculous because it is not induced by a cosimplicial map. Obviously $m \circ \mathcal{N}(\iota)=i d$.

Proposition 4.3. The miraculous map $m$ is a morphism in Cochehain.

Proof. Recall (see Lemma 3.12) that the differential acts on $(a, \sigma) \in C^{q} \times S_{q}$ by

$$
d^{q}(a, \sigma)=\sum_{0 \leq i \leq q+1}(-1)^{i}\left(d_{\bar{\sigma}(i)}(a), d_{i}(\sigma)\right)
$$

where $\bar{\sigma}:\{0, \ldots, q+1\} \rightarrow\{0, \ldots, q+1\}$ fixes $0, q+1$ and equals $\sigma$ on $\{1, \ldots, q\}$. The coboundary operators $d_{i}: S_{q} \rightarrow S_{q+1}$ are given by $d_{0}(\sigma):=\mathbb{1} \times \sigma, d_{q+1}(\sigma):=\sigma \times \mathbb{1}$ and $d_{i}(\sigma)$ is the permutation obtained by doubling the $i$ th input of $\sigma$. These coboundary operators were introduced in 18 , Example 12]. So, for $(a, \sigma) \in C^{q} \times S_{q}=F_{\mathcal{S}}\left(C^{\bullet}\right)^{q}$,

$$
m d^{q}(a, \sigma)=\sum_{0 \leq i \leq q+1}(-1)^{i} \operatorname{sgn}\left(d_{i}(\sigma)\right) d_{\bar{\sigma}(i)}(a)
$$

while

$$
d^{q} m(a, \sigma)=\sum_{0 \leq i \leq q+1}(-1)^{i} \operatorname{sgn}(\sigma) d_{i}(a)=\sum_{0 \leq i \leq q+1}(-1)^{\bar{\sigma}(i)} \operatorname{sgn}(\sigma) d_{\bar{\sigma}(i)}(a) .
$$

Comparing the terms at $d_{\bar{\sigma}(i)}(a)$ one sees that $m$ will be a cochain map if we prove that

$$
(-1)^{\bar{\sigma}(i)} \operatorname{sgn}(\sigma)=(-1)^{i} \operatorname{sgn}\left(d_{i}(\sigma)\right), \text { for } 0 \leq i \leq q+1
$$

The last equality can be established by a direct verification.

Theorem 4.4. Let $C^{\bullet}$ be a cosimplicial chain complex with finitely generated torsion-free components. Then

$$
\overline{\operatorname{Tot}}(\iota): \overline{\operatorname{Tot}}\left(C^{\bullet}\right) \rightarrow \overline{\operatorname{Tot}}\left(F_{\mathcal{S}}\left(C^{\bullet}\right)\right) \text { and }|m|: \overline{\operatorname{Tot}}\left(F_{\mathcal{S}}\left(C^{\bullet}\right)\right) \rightarrow \overline{\operatorname{Tot}}\left(C^{\bullet}\right)
$$

are natural mutually inverse homotopy equivalences. Moreover, $|m| \circ \overline{\operatorname{Tot}}(\iota)=i d$ and there is a natural cochain homotopy between $\overline{\operatorname{Tot}}(\iota) \circ|m|$ and $i d$.

[July 2012] 
Proof. The equation $|m| \circ \overline{\operatorname{Tot}}(\iota)=i d$ is trivial. Therefore it remains to prove the existence of a natural cochain homotopy between $\overline{\operatorname{Tot}}(\iota) \circ|m|$ and $i d$. We do it by applying Proposition A.5 of Appendix A.

For $A \in$ Chain, let $A^{\#}:=\underline{\operatorname{Chain}}(A, \mathbb{Z})$, where $\mathbb{Z}$ are the integers considered as the chain complex concentrated in degree 0 , denote the linear dual. The components $\left(A^{\#}\right)_{n}$ of the dual $A^{\#}$ are given by

$$
\left(A^{\#}\right)_{n}=A_{-n}^{\#}, \quad n \in \mathbb{Z}
$$

For $q \geq 0$ we denote by $\Delta[q]$ the standard simplicial $q$-simplex and $M[q]$ the simplicial abelian group spanned by $\Delta[q]$. Let $D[q]$ be the cosimplicial abelian group given as the componentwise linear dual of $M[q]$. We will interpret $M[q]$ (resp. $D[q]$ ) as a simplicial (resp. cosimplicial) chain complex concentrated in degree zero, with trivial vertical differentials.

Let $\mathrm{C} h \subset$ Chain be the subcategory consisting of chain complexes with finitely generated torsion-free components. To apply Proposition A.5 we put $\mathcal{D}=\mathcal{C} h^{\Delta}, A=B=\mathcal{N}\left(F_{\mathcal{S}}(-)\right)$, $f=\mathcal{N}(\iota) \circ m, g=i d$ and $\mathfrak{M}=\{D[0], D[1], D[2], \ldots\}$. We prove that $F_{\mathcal{S}}(-)^{q}$ is corepresented, with models $\mathfrak{M}$, in Lemma 4.5 below. It is also obvious that $(\mathcal{N}(\iota) \circ m)^{0}=i d^{0}$ and that $F_{\mathcal{S}}(D[q])^{\bullet}$ is concentrated in chain degree 0. The acyclicity of models is established in Proposition 4.8. Hence, all the conditions of Proposition A.5 are satisfied and we complete the proof of the theorem.

Lemma 4.5. The functor $F: \mathrm{Ch}^{\Delta} \rightarrow$ Chain defined by $F\left(C_{*}^{\bullet}\right):=F_{\mathcal{S}}\left(C_{*}^{\bullet}\right)^{q}$ is corepresented with the model $D[q]$.

Proof. Observe that the functor $F_{\delta}: \operatorname{Chain}^{\Delta} \rightarrow$ Chain $^{\Delta}$ takes objects from $\mathrm{Ch}^{\Delta}$ to the objects of $\mathrm{Ch}^{\Delta}$. Then the functor $F$ factorizes as a composite

$$
\mathcal{C} h^{\Delta} \stackrel{F_{s}}{\longrightarrow} \mathcal{C} h^{\Delta} \stackrel{e v_{q}}{\longrightarrow} \text { Chain. }
$$

Moreover, the full subcategory $\mathrm{C} h$ is the category of strongly dualizable objects in Chain. We conclude that $F$ is corepresented by Lemmas A.2 and A.3.

Analysis of $F_{\delta}(D[q])$. Let us describe $F_{\delta}(D[q])$ explicitly. To fix the notation, recall that $\Delta[q]$ is a simplicial set with

$$
\Delta[q]_{n}=\left\{\left(a_{0}, \ldots, a_{n}\right) ; 0 \leq a_{0} \leq \cdots \leq a_{n} \leq q\right\}, n \geq 0,
$$

the simplicial structure being given by

$$
\begin{aligned}
\partial_{i}\left(a_{0}, \ldots, a_{n}\right) & :=\left(a_{0}, \ldots, a_{i-1}, a_{i+1}, \ldots, a_{n}\right), \text { and } \\
s_{i}\left(a_{0}, \ldots, a_{n}\right) & :=\left(a_{0}, \ldots, a_{j}, a_{j}, \ldots, a_{n}\right), \text { for } 0 \leq i \leq n .
\end{aligned}
$$

The simplicial abelian group $M[q]$ generated by $\Delta[q]$ has the basis

$$
\left\{\left(a_{0}, \ldots, a_{n}\right) ; 0 \leq a_{0} \leq \cdots \leq a_{n} \leq q\right\}
$$


Let us denote by

$$
\left\{\left\langle a_{0}, \ldots, a_{n}\right\rangle ; 0 \leq a_{0} \leq \cdots \leq a_{n} \leq q\right\} .
$$

the dual basis of $D[q]^{n}$. The coboundary operators are then given by

$$
d_{i}\left\langle a_{0}, \ldots, a_{n}\right\rangle=\sum_{a_{i-1} \leq s \leq a_{i}}\left\langle a_{0}, \ldots, a_{i-1}, s, a_{i+1}, \ldots, a_{n}\right\rangle
$$

where we assume $a_{-1}:=0$ and $a_{n+1}:=q$ so the formula makes sense also for $i=0$ or $i=n+1$. For instance, for the generator $\langle 1,1\rangle \in D[3]^{1}$ we have

$$
d_{0}\langle 1,1\rangle=\langle 0,1,1\rangle+\langle 1,1,1\rangle, d_{1}\langle 1,1\rangle=\langle 1,1,1\rangle, d_{2}\langle 1,1\rangle=\langle 1,1,1\rangle+\langle 1,1,2\rangle+\langle 1,1,3\rangle .
$$

The above formulas show that $D[q]$ is not spanned by a cosimplicial set.

It is clear from the above explanation that elements of $F_{\mathcal{S}}(D[q])^{n}$ are linear combinations of the expressions of the form

$$
\left(\left\langle a_{0}, \ldots, a_{n}\right\rangle, \sigma\right), \text { for } 0 \leq a_{0} \leq \cdots \leq a_{n} \leq q, \sigma \in S_{n} .
$$

The differential in $\mathcal{N}\left(F_{\mathcal{S}}(D[q])\right)$ is given by the formula

$$
d^{n}\left(\left\langle a_{0}, \ldots, a_{n}\right\rangle, \sigma\right)=\sum_{0 \leq i \leq n+1}(-1)^{i}\left(d_{\bar{\sigma}(i)}\left\langle a_{0}, \ldots, a_{n}\right\rangle, d_{i}(\sigma)\right),
$$

where the definition of $\bar{\sigma}(i)$ and $d_{i}(\sigma)$ was recalled in the proof of Proposition 4.3 .

In [18, page 481] we introduced simple permutations whose definition we briefly recall. We defined first, for each $\sigma \in S_{n}$, a natural number $g(\sigma), 0 \leq g(\sigma) \leq n$, which we called the grade of $\sigma$, as follows. The grade of the unit $\mathbb{1}_{n} \in S_{n}$ is $n-1, g\left(\mathbb{1}_{n}\right):=n-1$. For $\sigma \neq \mathbb{1}_{n}$, let

$$
\begin{aligned}
a(\sigma) & :=\max \left\{i ; \sigma=\mathbb{1}_{i} \times \tau \text { for some } \tau \in S_{n-i}\right\}, \text { and } \\
c(\sigma) & :=\max \left\{j ; \sigma=\lambda \times \mathbb{1}_{j} \text { for some } \lambda \in S_{n-j}\right\} .
\end{aligned}
$$

There clearly exists a unique $\omega=\omega(\sigma) \in S_{n-a(\sigma)-c(\sigma)}$ such that $\sigma=\mathbb{1}_{a(\sigma)} \times \omega(\sigma) \times \mathbb{1}_{c(\sigma)}$. Let, finally, $b(\sigma)$ be the number of "doubled strings" in $\omega(\sigma)$,

$$
b(\sigma):=\{1 \leq s<k ; \omega(s+1)=\omega(s)+1\} .
$$

The grade of $\sigma$ was then defined by

$$
g(\sigma):=a(\sigma)+b(\sigma)+c(\sigma)
$$

see Figure 1 of [18] for examples. We called $\sigma \in S_{k}, k \geq 1$, simple if $g(\sigma)=0$.

Now, for each $\sigma \in S_{n}, \sigma \neq \mathbb{1}_{n}$, we define a unique simple $\kappa=\kappa(\sigma) \in S_{k}, k=n-g(\sigma)$, by contracting all "multiple strings" of $\omega(\sigma)$ into "simple" ones. We put $\kappa\left(\mathbb{1}_{n}\right):=\mathbb{1}_{1}$. Since, for $\sigma \in S_{n}$ and $0 \leq i \leq n+1, \kappa(\sigma)=\kappa\left(d_{i}(\sigma)\right)$, for each cosimplicial chain complex $C^{\bullet}$ one has the decomposition

$$
F_{\mathcal{S}}\left(C^{\bullet}\right)=\bigoplus_{\chi \text { simple }} F_{\mathcal{S}}^{\chi}\left(C^{\bullet}\right)
$$

[July 2012] 


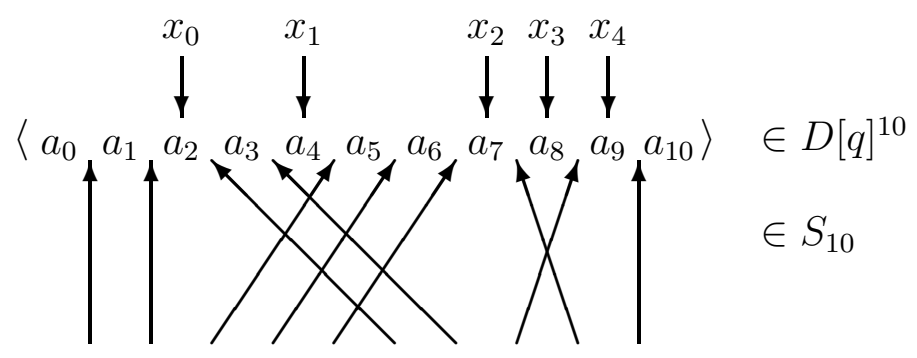

Figure 4. An element $(a, \sigma) \in F_{\delta}(D[q])^{10}$.

in which the subspaces

$$
F_{\mathcal{S}}^{\chi}\left(C^{\bullet}\right):=\left\{(a, \sigma) \in F_{\mathcal{S}}\left(C^{\bullet}\right) ; \kappa(\sigma)=\chi\right\}
$$

are invariant under coboundary operators. Let us see what happens if $C^{\bullet}=D[q]$.

Consider a simple permutation $\chi \in S_{m}$ different from $\mathbb{1}_{1} \in S_{1}$. We claim that there is a direct sum decomposition

$$
F_{\mathcal{S}}^{\chi}(D[q])=\bigoplus_{0 \leq x_{0} \leq \cdots \leq x_{m} \leq q} F_{\mathcal{S}}^{\chi}(D[q])_{x_{0}, \ldots, x_{m}},
$$

where $F_{\delta}^{\chi}(D[q])_{x_{0}, \ldots, x_{m}}$ is the smallest subspace of $F_{\mathcal{S}}(D[q])$ invariant under the coboundary operators and containing

$$
\left(\left\langle x_{0}, \ldots, x_{m}\right\rangle, \chi\right) \in F_{\delta}^{\chi}(D[q])^{m} .
$$

Before we formulate a formal argument that $F_{\delta}^{\chi}(D[q])$ indeed decomposes as in (21), we give the following example.

Example 4.6. Figure 4 symbolizes an element $(a, \sigma) \in F_{\mathcal{S}}(D[q])^{10}$, with $a=\left\langle a_{0}, \ldots, a_{10}\right\rangle$ and $\sigma \in$ $S_{10}$ the permutation determined by the arrows at the bottom of the picture. The corresponding simple permutation $\chi:=\kappa(\sigma)$ is

$$
\Varangle \searrow \in S_{4}
$$

and $(a, \sigma)$ belongs to the subspace $F_{\mathcal{S}}^{\chi}(D[q])_{x_{0}, \ldots, x_{4}}$ generated by

$$
\left\langle x_{0} \chi^{x_{1}} \chi^{x_{2}} \chi^{x_{3}} x^{x_{4}}\right\rangle \in F_{\mathcal{S}}(D[q])^{4},
$$

where $\left(x_{0}, x_{1}, x_{2}, x_{3}, x_{4}\right):=\left(a_{2}, a_{4}, a_{7}, a_{8}, a_{9}\right)$.

Let us describe how to decide to which subspace $F_{\delta}^{\chi}(D[q])_{x_{0}, \ldots, x_{m}}$ a general $(a, \sigma) \in F_{\mathcal{S}}(D[q])$ belongs. The permutation $\chi$ is determined easily, it is the simple permutation $\chi:=\kappa(\sigma) \in S_{m}$ associated to $\sigma$. If $a=\left\langle a_{0}, \ldots, a_{n}\right\rangle$, then $x_{0}, \ldots, x_{m}$ is the sequence obtained from $a_{0}, \ldots, a_{n}$ by deleting $a_{i}^{\prime} s$ located between doubled arrows of $\sigma$, between $\langle$ and a vertical arrow $\uparrow$, or between 
$\uparrow$ and $\rangle$. We believe that everything is clear from Figure 6 . Let finally $a_{\sigma}:=\left\langle x_{0}, \ldots, x_{m}\right\rangle$. It is easy to verify that then

$$
F_{\delta}^{\chi}(D[q])_{x_{0}, \ldots, x_{m}}=\left\{(a, \sigma) \in F_{\mathcal{S}}(D[q]) ; \kappa(\sigma)=\chi, a_{\sigma}=\left\langle x_{0}, \ldots, x_{m}\right\rangle\right\}
$$

which makes (21) obvious.

Let $\widehat{D}[q]$ be the augmented cosimplicial group obtained by adding to $D[q]$ the piece $\widehat{D}[q]^{-1}$ spanned by the empty bracket \langle\rangle and $d_{0}: \widehat{D}[q]^{-1} \rightarrow \widehat{D}[q]^{0}$ defined by

$$
d_{0}\langle\rangle:=\sum_{0 \leq s \leq q}\langle s\rangle
$$

We claim that, for each simple permutation $\chi \in S_{m}$ different from $\mathbb{1} \in S_{1}$, there is an isomorphisms of cochain complexes

$$
\mathcal{N}\left(F_{\mathcal{S}}^{\chi}(D[q])_{x_{0}, \ldots, x_{m}}\right) \cong \uparrow^{2 m+2} \mathcal{N}\left(\widehat{D}\left[x_{0}\right]\right) \otimes \mathcal{N}\left(\widehat{D}\left[x_{1}-x_{0}\right]\right) \otimes \cdots \otimes \mathcal{N}\left(\widehat{D}\left[x_{m}-x_{m-1}\right]\right) \otimes \mathcal{N}\left(\widehat{D}\left[q-x_{m}\right]\right)
$$

where $\uparrow^{2 m+2}$ in the right hand side means that the degrees are shifted up by $2 m+2$. For instance, to $(a, \sigma) \in F_{\mathcal{S}}(D[q])^{10}$ in Figure 4 it corresponds

$$
\begin{aligned}
& \left\langle a_{0}, a_{1}\right\rangle \otimes\left\langle a_{3}\right\rangle \otimes\left\langle a_{5}, a_{6}\right\rangle \otimes\langle\rangle \otimes\langle\rangle \otimes\left\langle a_{10}\right\rangle \in \\
& \widehat{D}\left[a_{2}\right]^{1} \otimes \widehat{D}\left[a_{4}-a_{2}\right]^{0} \otimes \widehat{D}\left[a_{7}-a_{4}\right]^{1} \otimes \widehat{D}\left[a_{8}-a_{7}\right]^{-1} \otimes \widehat{D}\left[a_{9}-a_{8}\right]^{-1} \otimes \widehat{D}\left[q-a_{9}\right]^{0} .
\end{aligned}
$$

Isomorphism (22) is defined as follows: to each $\left(\left\langle a_{0}, \ldots, a_{n}\right\rangle, \sigma\right) \in F_{\mathcal{S}}^{\chi}(D[q])_{x_{0}, \ldots, x_{m}}^{n}$ one associates the element of the tensor product in the right hand side obtained by replacing, in $\left\langle a_{0}, \ldots, a_{n}\right\rangle$, each $x_{j} \in\left\{x_{0}, \ldots, x_{m}\right\}$ by $\rangle \otimes\langle$.

Combining isomorphisms (20), (21) and (22) with the obvious equality $F_{\mathcal{S}}^{\mathbb{1}}(D[q]) \cong D[q]$, we finally get:

Lemma 4.7. For each $q \geq 1$, one has the isomorphism of cochain complexes:

$$
\begin{aligned}
& \mathcal{N}\left(F_{\mathcal{S}}(D[q])\right) \cong \\
& \quad \cong \mathcal{N}(D[q]) \oplus \bigoplus \uparrow^{2 m+2} \mathcal{N}\left(\widehat{D}\left[x_{0}\right]\right) \otimes \mathcal{N}\left(\widehat{D}\left[x_{1}-x_{0}\right]\right) \otimes \cdots \otimes \mathcal{N}\left(\widehat{D}\left[x_{m}-x_{m-1}\right]\right) \otimes \mathcal{N}\left(\widehat{D}\left[q-x_{m}\right]\right)
\end{aligned}
$$

where the summation in the right hand side is taken over all simple permutations $\chi \in S_{m}$ different from $\mathbb{1} \in S_{1}$ and all $0 \leq x_{0} \leq \cdots \leq x_{m} \leq q$.

Proposition 4.8. For each $q \geq 0, H^{\geq 1}\left(\mathcal{N}\left(F_{\mathcal{S}}(D[q])\right)\right)=0$.

Proof. It is a standard fact that $H^{i}(\mathcal{N}(D[q]))=0$ for all $i \geq 1$ and that $H^{i}(\mathcal{N}(\widehat{D}[q]))=0$ for all $i$. The proposition then follows from Lemma 4.7 and the Künneth theorem. 


\section{Bar construction And proof of Theorem C}

In this section we analyze the bar construction of an associative algebra and the induced structures on the Hochschild complex. We then formulate Proposition 5.1 relating the operads $\mathcal{B}$ and $\mathcal{T}$, and prove Theorem $\mathrm{C}$.

As everywhere in this paper, we work over the ring $\mathbb{Z}$ of integers, though all results and definitions in this section remain valid over an arbitrary commutative unital ground ring $R$, only "abelian group" must be translated into " $R$-module." Let $B_{*}^{A-A}(A)=B_{*}(A, A, A)$ be the twosided bar construction of a unital associative algebra $A=(A, \mu, 1)$ so that $B_{n}^{A-A}(A)=A \otimes A^{\otimes n} \otimes A$ is the free $A$-bimodule on $A^{\otimes n}, n \geq 0$. The bar construction extends into a functor $B_{*}^{A-A}(A)$ : $\mathcal{J} S \rightarrow A$-biMod from the crossed interval group $\mathcal{J} S$ introduced in Definition 3.4 to the category $A$-biMod of $A$-bimodules as follows.

On objects, $B_{*}^{A-A}(A)(\langle n\rangle):=B_{n+1}^{A-A}(A)$. For $a_{-1} \otimes a_{0} \otimes \cdots \otimes a_{m} \otimes a_{m+1} \in B_{m+1}^{A-A}(A)$ and a morphism $f:\langle m\rangle \rightarrow\langle n\rangle$ in $\mathcal{J} S$ put

$$
B_{*}^{A-A}(A)(f)\left(a_{-1} \otimes a_{0} \otimes \cdots \otimes a_{m} \otimes a_{m+1}\right):=\bar{a}_{-1} \otimes \bar{a}_{0} \otimes \cdots \otimes \bar{a}_{n} \otimes \bar{a}_{n+1} \in B_{n+1}^{A-A}(A),
$$

where $\bar{a}_{i}$ is, for $-1 \leq i \leq n+1$, the product of $a_{j}$ 's, $j \in f^{-1}(i)$, in the order specified by the linear order on the fiber $f^{-1}(i)$. If $f^{-1}(i)=\emptyset$ we put $\bar{a}_{i}:=1$.

It is easy to see that the composition $\Delta^{\text {op }} \stackrel{\text { joy }}{\cong} \mathcal{J} \hookrightarrow \mathcal{J} S \stackrel{B_{*}^{A-A}(A)}{\longrightarrow} A$-biMod is the standard simplicial $A$-A-bimodular structure of the bar construction. The functor $B_{*}^{A-A}(A): \mathcal{J} S \rightarrow A$-biMod is in fact induced from the symmetric bar construction $B_{*}^{\text {sym }}(A): \Delta S \rightarrow \mathcal{A} b$ introduced in [6], by the diagram

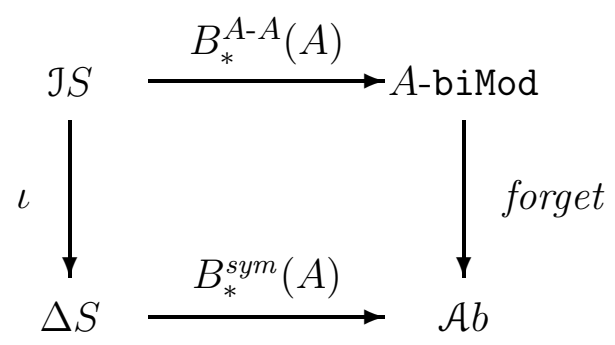

in which $\iota$ is the inclusion (17) and $\mathcal{A} b$ the category of abelian groups.

Consequently, for any $A$-bimodule $M$, the space $C^{*}(A ; M):=\operatorname{Hom}_{A-A}\left(B_{*}^{A-A}(A), M\right) \cong$ $\operatorname{Lin}\left(A^{\otimes *}, M\right)$ of Hochschild cochains of $A$ with coefficients in $M$ is an $(\mathcal{J} S)^{\text {op }}$-abelian group. This structure will be crucial for us.

Let us turn our attention to the $\mathbb{N}$-coloured operad $B$ from Section 1. As we already noted, the spaces $B_{k}^{l}$ (i.e. $B_{k_{1}, \ldots, k_{n}}^{l}$ with $n=1$ and $k=k_{1}$ ) are the Hom-sets of the underlying category $\mathcal{U}(B)$ of this coloured operad. It turns out that this category is in fact a linearization of $(\mathcal{J} S)^{\mathrm{op}}$, by which we mean that

$$
\operatorname{Span}\left(\mathcal{J} S(\langle l-1\rangle,\langle k-1\rangle) \stackrel{\cong}{\longrightarrow} B_{k}^{l}\right.
$$




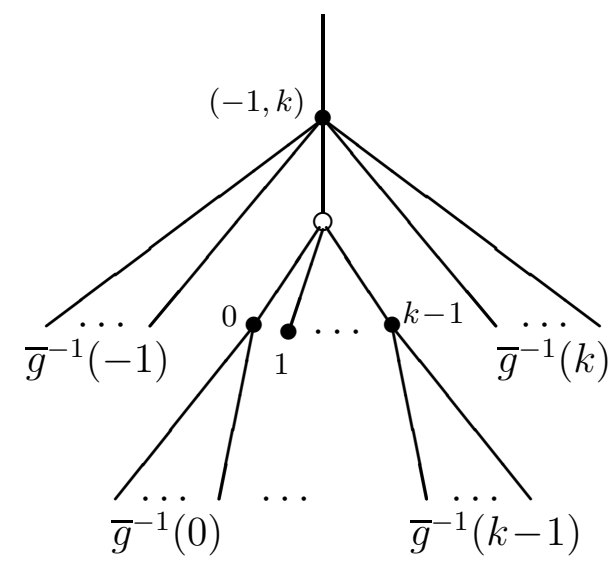

FiguRE 5 . The $(l ; k)$-tree representing the operation $O_{g} \in B_{k}^{l}$. It has one white vertex and $k+1$ black vertices. To each black vertex one attaches legs as indicated in the picture, labeled by the elements of the fibers of $\bar{g}$. If the corresponding fiber is empty, the black vertex becomes a special one - in the above picture this is the vertex labeled 1.

for each $k, l \geq 0$, where $\operatorname{Span}(-)$ denotes the free abelian group functor.

To describe the above isomorphism, it will be convenient to interpret elements of $B$ as operations on the Hochschild cochain complex of $A$, so we assume that $A$ is generic. The isomorphism is then induced by the set-map that assigns to each morphism $g:\langle l-1\rangle \rightarrow\langle k-1\rangle$ in $\mathcal{J} S$ the natural operation $O_{g}: C^{k}(A ; A) \rightarrow C^{l}(A ; A) \in B_{k}^{l}$ constructed as follows.

Let $\bar{g}:\{0, \ldots, l-1\} \rightarrow\{-1, \ldots, k\}=\langle k-1\rangle$ be the restriction $\bar{g}:=\left.g\right|_{\{0, \ldots, l-1\}}$ and denote, for each $-1 \leq i \leq k$ for which $\bar{g}^{-1}(i)$ is non-empty

$$
\bar{g}^{-1}(i)=\left(\sigma_{1}^{i}, \ldots, \sigma_{s(i)}^{i}\right),
$$

where the numbers $\sigma_{1}^{i}, \ldots, \sigma_{s(i)}^{i} \in\{0, \ldots, l-1\}$ are ordered according to the restriction of the order of the fiber $g^{-1}(i)$ to its subset $\bar{g}^{-1}(i) \subset g^{-1}(i)$.

For $f: A^{\otimes k} \rightarrow A \in C^{k}(A ; A)$ the cochain $O_{g}(f): A^{\otimes l} \rightarrow A \in C^{l}(A ; A)$ is given by the formula

$$
O_{g}(f)\left(a_{0}, \ldots, a_{l-1}\right)=\bar{a}_{-1} \cdot f\left(\bar{a}_{0}, \ldots, \bar{a}_{k-1}\right) \cdot \bar{a}_{k},
$$

where, for $a_{0}, \ldots, a_{l-1} \in A$ and $i \in\{-1, \ldots, k\}$,

$$
\bar{a}_{i}:= \begin{cases}a_{\sigma_{1}^{i}} \cdot \ldots \cdot a_{\sigma_{s(i)}^{i}}, & \text { if } \bar{g}^{-1}(i) \neq \emptyset, \text { and } \\ 1, & \text { otherwise. }\end{cases}
$$

The $(l ; k)$-tree encoding the operation $O_{g}$ is depicted in Figure 5 .

It follows from general properties of coloured operads that $B_{k}^{l}$ acts on $B_{k_{1}, \ldots, k_{n}}^{l}$ covariantly on the upper index and contravariantly on each lower index. Therefore, the spaces $B_{k_{1}, \ldots, k_{n}}^{l}$ assemble into a functor

$$
\mathcal{B}_{\bullet_{1}, \ldots, \bullet_{n}}:(\mathcal{J} S)^{\mathrm{op}} \times(\mathcal{J} S)^{n} \rightarrow \mathcal{A} b
$$


Precomposing this functor with the canonical functor

$$
\Delta \times\left(\Delta^{\mathrm{op}}\right)^{n} \rightarrow(\mathcal{J} S)^{\mathrm{op}} \times(\mathcal{J} S)^{n}
$$

we get the functor $B_{\bullet_{1}, \ldots, \bullet_{n}}$ in (4).

Proposition 5.1. The functor $\mathcal{B}_{\bullet_{1}, \ldots, \bullet_{n}}:(\mathcal{J} S)^{\mathrm{op}} \times(\mathcal{J} S)^{n} \rightarrow \mathcal{A} b$ is the result of application of the functor $F_{\mathcal{S}}$ described in Lemma 3.12 to the functor $T_{\bullet_{1}, \ldots, \bullet_{n}}$. Therefore,

$$
\mathcal{B}=\overline{\operatorname{Tot}}\left(\underline{\operatorname{Tot}}\left(F_{\delta} T_{\bullet_{1}, \ldots, \bullet_{n}}^{\bullet}\right)\right)=\overline{\operatorname{Tot}}\left(F_{\delta} \underline{\operatorname{Tot}}\left(T_{\bullet_{1}, \ldots, \bullet_{n}}^{\bullet}\right)\right),
$$

and the inclusion $\mathcal{T} \rightarrow \mathcal{B}$ is induced by the unit of the monad $F_{\mathcal{s}}$. The same relationship holds between $\widehat{\mathcal{T}}$ and $\widehat{\mathcal{B}}$.

Proof. The proposition easily follows from the explicit description of $\mathcal{B}$ and $\mathcal{T}$ given on pages $7-9$ of Section 1 .

We can finally give

Proof of Theorem $C$. The inclusion $\mathcal{T} \hookrightarrow \mathcal{B}$ is a weak equivalence by Theorem 4.4 and Proposition 5.1. It is shown in the proof of [2, Theorem 5.9] that the inclusion $\mathcal{B} r \hookrightarrow \mathcal{T}$ is a weak equivalence, too. All vertical projections $\mathcal{B} \rightarrow \operatorname{Nor}(\mathcal{B}), \mathcal{T} \rightarrow \operatorname{Nor}(\mathcal{T})$ and $\mathcal{B} r \rightarrow \operatorname{Nor}(\mathcal{B} r)$ are weak equivalences because they are normalization maps. So, all morphisms in the two upper triangles and between them are weak equivalences by two out of three property, which gives (2).

To establish the homotopy type of the operads in the upper two triangles of Figure 3, it is, by (2), enough to establish the homotopy type of one of them.

The fact that $\mathcal{T}$ has the homotopy type of $C_{-*}(\mathcal{D})$ follows from [1]. It was shown, in the proof of Proposition 2.14 of that paper, that the operad $T$ (denoted $\mathcal{O}$ there) is isomorphic to the second filtration $\mathcal{L}_{(2)}$ of the lattice path operad $\mathcal{L}$. Theorem 3.8 of $\mathbb{1}$ then claims that the totalization of $\mathcal{L}_{(2)}$, isomorphic to $\mathcal{T}$ due to the isomorphism $T \cong \mathcal{L}_{(2)}$, is an $E_{2}$-operad in Chain.

Item (4) of the theorem is obvious from the analysis in Subsection 1.8 which immediately implies that the inclusion $\widehat{\mathcal{B}} \hookrightarrow \mathcal{B}$ induces homology isomorphisms $\widehat{\mathcal{B}}(n) \sim \mathcal{B}(n)$ for each $n \geq 1$. The same relationship holds also for the suboperads $\widehat{\mathcal{T}} \subset \mathcal{T}$ and $\widehat{\mathcal{B} r} \subset \mathcal{B} r$.

Regarding the homotopy type of the operads in the bottom triangle of Figure 3, it suffices, given (4), to prove that the dg-abelian groups $\widehat{\mathcal{B}}(0)$ and $\widehat{\mathcal{T}}(0)$ are acyclic, because $\widehat{\mathcal{B} r}(0)=0$ by definition. This was done in [18, Example 12]. 


\section{Appendix A. Acyclic Models Theorem for cochain functors.}

We introduce a theory of acyclic models for functors with values in cochain complexes (cochain functors). Due to the lack of a suitable concept of cofree modules, this theory is not merely a dualization of the chain version.

We present the necessary notions and results in a general setting with an arbitrary complete, cocomplete closed symmetric monoidal category $V$ as the underlying category, although $V$ will, for the purposes of this paper, always be the category of chain complexes Chain. We choose this more categorical approach because, in our opinion, it makes our definitions clearer in the special case $V=$ Chain.

We refer to the book [13] for definitions of a category, functor and natural transformation enriched in a closed symmetric monoidal category, as well as for a discussion concerning the difference between enriched and nonenriched natural transformations. In particular, we remind that there can exist natural transformations between $V$-functors, which are not $V$-transformations.

Let $(V, \otimes, I)$ be a complete and cocomplete closed symmetric monoidal category. Let $\mathcal{D}$ be a $V$-category. Let $F: \mathcal{D} \rightarrow V$ be a $V$-functor and $\mathfrak{M}$ a set of objects of $\mathcal{D}$. Let us denote, for $X \in \mathcal{D}$, by $\widetilde{F}(X)$ the following object of $V$ :

$$
\widetilde{F}(X):=\underline{V}\left(\coprod_{M \in \mathfrak{M}} \underline{\mathcal{D}}(X, M), F(M)\right)=\prod_{M \in \mathfrak{M}} \underline{V}(\underline{\mathcal{D}}(X, M), F(M)) .
$$

In the above display, as well as in the rest of this section, we used the notation $\underline{\mathcal{e}}(-,-)$ for the enriched Hom-functor of a $V$-category $\mathcal{C}$.

It is obvious that (23) defines a $V$-functor $\widetilde{F}: \mathcal{D} \rightarrow V$. There exists the canonical $V$-enriched transformation $\lambda: F \rightarrow \widetilde{F}$ such that, for $X \in \mathcal{D}$ and $M \in \mathfrak{M}$, the component of $\lambda$

$$
\lambda(X): F(X) \rightarrow \underline{V}(\underline{\mathcal{D}}(X, M), F(M))
$$

is the morphism in $V$ which corresponds under adjunction to the evaluation morphism

$$
F(K) \otimes \underline{\mathcal{D}}(X, M) \stackrel{1 \otimes F}{\longrightarrow} F(X) \otimes \underline{V}(F(X), F(M)) \rightarrow F(M) .
$$

Definition A.1. We say that $F: \mathcal{D} \rightarrow V$ as above is corepresented with models $\mathfrak{M}$ if there exists a (not necessarily $V$-enriched) transformation $\chi: \widetilde{F} \rightarrow F$ such that $\chi \lambda=i d_{F}$.

Recall [20, page 173] that an object $K \in V$ is called strongly dualizable if the morphism

$$
\nu: K^{\#} \otimes K \rightarrow \underline{V}(K, K)
$$

is an isomorphism. Here, $K^{\#}:=\underline{V}(K, I)$ and $\nu$ is adjoint to the morphism

$$
\underline{V}(K, I) \rightarrow \underline{V}(K \otimes K, I \otimes K) \simeq \underline{V}(K \otimes K, K) .
$$


Let $\mathcal{C}$ be a small $V$-enriched category. Assume that, for any two $a, b \in \mathcal{C}$, the object $\underline{\mathcal{C}}(a, b) \in V$ is strongly dualizable. Let $C_{q}, q \in \mathcal{C}$, be a representable presheaf i.e. the functor $C_{q}$ : $\mathrm{Cop}^{\rightarrow} V$ given by $C_{q}(b):=\underline{\mathfrak{e}}(b, q)$, for $b \in \mathcal{C}$. Let, finally, $D_{q}: \mathcal{C} \rightarrow V$ be the functor defined as $D_{q}(b):=C_{q}^{\#}(b)$.

Let Dual $\subset V$ be the full subcategory of strongly dualizable objects and Dual ${ }^{\mathrm{e}}$ the enriched category of enriched functors $\mathcal{C} \rightarrow$ Dual and their enriched natural transformations. Let $q$ be an object of $\mathcal{C}$. An ample source of examples of corepresented functors is provided by the following lemma.

Lemma A.2. Let $q \in \mathcal{C}$. The enriched functor $e v_{q}: D u a l^{\mathcal{C}} \rightarrow V$, ev $v_{q}(X):=X(q)$, is corepresented with the model $D_{q}$.

Proof. Observe that dualization functor $(-)^{\#}$ induces a contravariant isomorphism of categories Dual ${ }^{\mathrm{e}} \cong D u a l^{\mathrm{eop}}$. Then

$$
\begin{aligned}
& \widetilde{e v_{q}}(X)=\underline{V}\left(\underline{D u a l} l^{\complement}\left(X, D_{q}\right), D_{q}(q)\right) \cong \underline{V}\left(\underline{D u a l}{ }^{\mathfrak{e}^{o p}}\left(D_{q}^{\#}, X^{\#}\right), \underline{\mathrm{e}}(q, q)^{\#}\right) \\
& \cong \underline{V}\left(\underline{D u a l}{ }^{\mathrm{Cop}}\left(C_{q}, X^{\#}\right), \underline{\mathrm{e}}(q, q)^{\#}\right) \cong \underline{V}\left(X^{\#}(q), \underline{\mathrm{e}}(q, q)^{\#}\right) \\
& \cong \underline{V}(\underline{\mathrm{e}}(q, q), X(q)) \text {. }
\end{aligned}
$$

Then we define $\chi: \widetilde{e v_{q}}(X) \rightarrow e v_{q}(X)$ by applying $\underline{V}(-, X(q))$ to the unit morphism $I \rightarrow \underline{\mathrm{e}}(q, q)$

$$
\underline{V}(\underline{\mathfrak{e}}(q, q), X(q)) \rightarrow \underline{V}(I, X(q)) \cong X(q)=e v_{q}(X) .
$$

The equation $\chi \lambda=i d_{e v_{q}}$ is obvious.

The following lemma together with Lemma A.2 allows to construct new corepresented functors.

Lemma A.3. Let $F: \mathcal{D} \rightarrow V$ be corepresented with models $\mathfrak{M}$ and let $A: \mathcal{D} \rightarrow \mathcal{D}$ be an endofunctor. Then the composite $F \circ A: \mathcal{D} \rightarrow V$ is corepresented with models $\mathfrak{M}$.

Proof. By direct verification we establish the equality $(\widetilde{F \circ A})(X)=\widetilde{F}(A(X))$. So we have that the composite

$$
F \circ A(X) \rightarrow(\widetilde{F \circ A})(X) \rightarrow F \circ A(X)
$$

equals the identity.

For an abelian closed symmetric monoidal category $V$ the category Coch $V$ of cochain complexes in $V$ has a canonical $V$-enrichment. The following theorem is a dual version of the classical Acyclic Models Theorem (see, for instance, [21, Theorem 28.3]). With our definitions in place, the arguments are standard so we leave the proof of this theorem as an exercise.

Theorem A.4. Let $V$ be an abelian symmetric monoidal closed category. Let $\mathcal{D}$ be a $V$-category and Coch $V$ the category of cochain complexes in $V$. Let $A^{*}, B^{*}: \mathcal{D} \rightarrow$ Coch $V$ be $V$-functors and $f^{*}, g^{*}: A^{*} \rightarrow B^{*}$ natural transformations such that $f^{0}=g^{0}$. Suppose that 
(i) $B^{q}: \mathcal{D} \rightarrow V$ is corepresented with models $\mathfrak{M}$, for each $q \geq 1$,

(ii) for $m \geq 1$ and each $M \in \mathfrak{M}$ the short exact sequence

$$
0 \rightarrow \operatorname{Ker}\left(d^{m}\right) \rightarrow A^{m}(M) \stackrel{d^{m}}{\rightarrow} \operatorname{Im}\left(d^{m}\right) \rightarrow 0
$$

splits, and

(iii) $H^{\geq 1}\left(A^{*}(M), d\right)=0$, for each $M \in \mathfrak{M}$.

Then there exists a natural cochain homotopy $H^{*}: f^{*} \sim g^{*}$.

When $V=$ Chain, Theorem A.4 implies:

Proposition A.5. Let $\mathcal{D}$ be a dg-category and CochChain the category of cochain complexes of chain complexes. Let $A_{*}^{*}, B_{*}^{*}: \mathcal{D} \rightarrow$ CochChain be dg-functors and $f_{*}^{*}, g_{*}^{*}: A_{*}^{*} \rightarrow B_{*}^{*}$ (not necessarily dg-enriched) transformations such that $f_{*}^{0}=g_{*}^{0}$. Suppose moreover that

(i) $B_{*}^{q}: \mathcal{D} \rightarrow$ Chain is corepresented with models $\mathfrak{M}$, for each $q \geq 1$,

(ii) $A_{*}^{q}(M)$ is a finitely generated torsion-free chain complex concentrated in degree 0 , for each $q \geq 1, M \in \mathfrak{M}$, and

(iii) $H^{\geq 1}\left(A_{0}^{*}(M), d\right)=0$, for each $M \in \mathfrak{M}$.

Then there exists a natural cochain homotopy $H_{*}^{*}: f_{*}^{*} \sim g_{*}^{*}$ that commutes with the vertical (chain) differentials and determines, for each $K \in \mathcal{D}$, a natural cochain homotopy between the induced maps $\left|f_{*}^{*}\right|^{*},\left|g_{*}^{*}\right|^{*}:\left|A_{*}^{*}(K)\right|^{*} \rightarrow\left|B_{*}^{*}(K)\right|^{*}$.

Proof. The only issue that has to be verified is that (ii) of Proposition A.5 implies (ii) of Theorem A.4, which is simple. The rest is obvious.

\section{Appendix B. Generic algebras}

This section is devoted solely to our proof of the following statement.

Theorem B.1. The free associative unital algebra $U:=\mathbb{T}\left(x_{1}, x_{2}, x_{3}, \ldots\right)$ generated by countably many generators $x_{1}, x_{2}, x_{3}, \ldots$ is generic.

The theorem will follow from Lemma B.2 below. The underlying abelian group of $U$ is free, with a preferred basis given by monomials. For $e \in U$ and a monomial $h$ we denote by $\left.e\right|_{h} \in \mathbb{Z}$ the coefficient at $h$ in this preferred basis.

Lemma B.2. For each $\left(l ; k_{1}, \ldots, k_{n}\right)$-tree $T$ there exist cochains $f_{i}^{T} \in C^{k_{i}}(U, U), 1 \leq i \leq n$, and a monomial $h^{T} \in U$ such that, for any $\left(l ; k_{1}, \ldots, k_{n}\right)$-tree $S$

$$
\left.O_{S}\left(f_{1}^{T}, \ldots, f_{n}^{T}\right)\left(x_{1}, \ldots, x_{l}\right)\right|_{h^{T}}= \begin{cases}1, & \text { if } S=T \\ 0, & \text { otherwise. }\end{cases}
$$

[July 2012] 
Proof. The cochains $f_{i}^{T}: U^{\otimes k_{i}} \rightarrow U, 1 \leq i \leq n$, will be determined by a choice of monomials $u_{1}^{i}, \ldots, u_{k_{i}}^{i} \in U$ by requiring that

$$
f_{i}^{T}\left(u_{1}^{i}, \ldots, u_{k_{i}}^{i}\right):=x_{l+i}, 1 \leq i \leq n,
$$

while $f_{i}^{T}\left(v_{1}, \ldots, v_{k_{i}}\right)=0$ for all monomials $v_{1}, \ldots, v_{k_{i}} \in U$ such that $\left(v_{1}, \ldots, v_{k_{i}}\right) \neq\left(u_{1}^{i}, \ldots, u_{k_{i}}^{i}\right)$ in $U^{\otimes k_{i}}$. We call $x_{l+i+1}$ the value of $f_{i}$.

The $j$ th member $u_{j}^{i}, 1 \leq j \leq k_{i}$, of the sequence $u_{1}^{i}, \ldots, u_{k_{i}}^{i}$ is defined as follows. Let $m_{j}^{i}$ be the $j$ th input of $f_{i}^{T}$ in the expression $O_{T}\left(f_{1}^{T}, \ldots, f_{n}^{T}\right)\left(x_{1}, \ldots, x_{l}\right)$. Clearly, $m_{j}^{i}$ is either 1 or a string made of some $x_{s}, 1 \leq s \leq l$ and/or of some $f_{t}^{T}(-, \ldots,-), 1 \leq t \leq n$. Then $u_{j}^{i}$ is given by replacing all function symbols in $m_{j}^{i}$ by their values, i.e. by replacing $f_{t}^{T}(-, \ldots,-)$ by $x_{l+t}$. The monomial $h^{T}$ is the result of the same procedure applied to $O_{T}\left(f_{1}^{T}, \ldots, f_{n}^{T}\right)\left(x_{1}, \ldots, x_{l}\right)$.

It is obvious that the cochains $f_{1}^{T}, \ldots, f_{n}^{T}$ and the monomial $h^{T}$ have the required property, because the tree $T$ can be uniquely reconstructed from them. This finishes the proof of the lemma.

Let us illustrate the above construction on the $(8 ; 3,3,1,3)$-tree in Figure 1. Since $l=8$, the values of $f_{1}^{T}, f_{2}^{T}, f_{3}^{T}, f_{4}^{T}$ are $x_{9}, x_{10}, x_{11}, x_{12}$, respectively. One has

$$
O_{T}\left(f_{1}^{T}, f_{2}^{T}, f_{3}^{T}, f_{4}^{T}\right)\left(x_{1}, \ldots, x_{8}\right)=x_{3} f_{1}^{T}\left(f_{2}^{T}\left(x_{5} x_{6}, 1, x_{8}\right), x_{1}, f_{3}^{T}\left(x_{7}\right)\right) f_{4}^{T}\left(x_{4}, 1, x_{2}\right) .
$$

The monomials $u_{1}^{1}, u_{2}^{1}$ and $u_{3}^{1}$ are obtained from the inputs $m_{1}^{1}=f_{2}^{T}\left(x_{5} x_{6}, 1, x_{8}\right), m_{2}^{1}=x_{1}$ and $m_{3}^{1}=f_{3}^{T}\left(x_{7}\right)$ of $f_{1}^{T}$ by replacing all function symbols by their values, so $\left(u_{1}^{1}, u_{2}^{1}, u_{3}^{1}\right)=\left(x_{10}, x_{1}, x_{11}\right)$. Therefore $f_{1}^{T}$ is defined by $f_{1}^{T}\left(x_{10}, x_{1}, x_{11}\right):=x_{9}$. Similarly, one gets

$$
f_{2}^{T}\left(x_{5} x_{6}, 1, x_{8}\right):=x_{10}, f_{3}^{T}\left(x_{7}\right):=x_{11}, f_{4}^{T}\left(x_{4}, 1, x_{2}\right):=x_{12} \text { and } h^{T}:=x_{3} x_{9} x_{12} .
$$

Proof of Theorem B.1. Since $\omega_{A}$ is epi, we only need to prove that, for $l, k_{1}, \ldots, k_{n} \geq 0$ and a linear combination $\sum_{t=1}^{s} \alpha_{t} T^{t}$ of $\left(l ; k_{1}, \ldots, k_{n}\right)$-trees, $\omega_{A}\left(\sum_{t=1}^{s} \alpha_{t} T^{t}\right)=0$ implies that $\alpha_{1}, \ldots, \alpha_{s}=$ 0 . For an arbitrary $r, 1 \leq r \leq s$, let $f_{1}^{T^{r}}, \ldots, f_{n}^{T^{r}}$ and $h^{T^{r}}$ be as in Lemma B.2 Then

$$
\begin{aligned}
0 & =\left.\omega_{A}\left(\sum_{t=1}^{s} \alpha_{t} T^{t}\right)\left(f_{1}^{T^{r}}, \ldots, f_{n}^{T^{r}}\right)\left(x_{1}, \ldots, x_{n}\right)\right|_{h^{T^{r}}} \\
& =\left.\sum_{t=1}^{s} \alpha_{t} O_{T^{t}}\left(f_{1}^{T^{r}}, \ldots, f_{n}^{T^{r}}\right)\left(x_{1}, \ldots, x_{n}\right)\right|_{h^{T^{r}}}=\alpha_{r} .
\end{aligned}
$$

Therefore $\alpha_{r}=0$ for each $1 \leq r \leq s$.

Problem. Give a characterization of generic algebras. We leave as an exercise to prove that, for instance, each algebra $A$ which contains $U$ as a subalgebra and a direct summand is generic. It is also clear that $U$ modulo the ideal generated by $x_{i}^{2}, i \geq 1$, is generic. Does there exists a 'minimal' (in an appropriate sense) generic algebra? 


\section{REFERENCES}

[1] M.A. Batanin and C. Berger. The lattice path operad and Hochschild cochains. In Proceedings of the 'Alpine Perspective on Algebraic Topology,' volume 504 of Contemp. Math., pages 23-52, 2009.

[2] M.A. Batanin, C. Berger, and M. Markl. Operads of natural operations I: Lattice paths, braces and Hochschild cochains. To appear in Actes de la Conférence "Operads 2009," arXiv:0906.4097, 2009.

[3] C. Berger and B. Fresse. Combinatorial operad actions on cochains. Math. Proc. Cambridge Philos. Soc., 137(1):135-174, 2004.

[4] B. Day and R. Street. Abstract substitution in enriched categories. J. Pure Appl. Algebra 179 (2003) 49-63.

[5] P. Deligne. A letter to Stasheff, Gerstenhaber, May, Schechtman and Drinfel'd. Unpublished, 1993.

[6] Z. Fiedorowicz. The symmetric bar construction. Preprint, available from the author's home page.

[7] Z. Fiedorowicz and J.-L. Loday. Crossed simplicial groups and their associated homology. Trans. Amer. Math. Soc., 326(1):57-87, 1991.

[8] P. Gabriel and M. Zisman. Calculus of Fractions and Homotopy Theory. Springer-Verlag, Berlin, Heidelberg, New York, 1967.

[9] M. Gerstenhaber and A. Voronov. Homotopy G-algebras and moduli space operad. I. Math. Res. Notices, 3 (1995), 141-153.

[10] V. Ginzburg and M.M. Kapranov. Koszul duality for operads. Duke Math. J., 76(1):203-272, 1994.

[11] A. Joyal. Disks, duality and $\Theta$-categories. Preprint, September 1997.

[12] R.M. Kaufmann. On spineless cacti, Deligne's conjecture and Connes-Kreimer's Hopf algebra. Topology, 46(1):39-88, 2007.

[13] G.M. Kelly, Basic concepts of enriched category theory. London Mathematical Society Lecture Note Series, $64,1982$.

[14] M. Kontsevich and Y. Soibelman. Deformations of algebras over operads and the Deligne conjecture. In Dito, G. et al., editor, Conférence Moshé Flato 1999: Quantization, deformation, and symmetries, number 21 in Math. Phys. Stud., pages 255-307. Kluwer Academic Publishers, 2000.

[15] R. Krasauskas. Skew-simplicial groups. Litovsk. Mat. Sb., 27:89-99, 1987.

[16] M. Makkai and M.Zawadovski. Duality for Simple $\omega$-Categories and Disks. TAC, 8(7):114-243, 2001.

[17] M. Markl. Handbook of Algebra, volume 5, chapter Operads and PROPs, pages 87-140. Elsevier, 2008.

[18] M. Markl. Cohomology operations and the Deligne conjecture. Czechoslovak Math. J., 132(57):473-503, 2007.

[19] M. Markl, S. Shnider, and J. D. Stasheff. Operads in Algebra, Topology and Physics, volume 96 of Mathematical Surveys and Monographs. American Mathematical Society, Providence, Rhode Island, 2002.

[20] J. P. May. Equivariant homotopy and cohomology theory, volume 91 of CBMS Regional Conference Series in Mathematics. Published for the Conference Board of the Mathematical Sciences, Washington, DC, 1996. With contributions by M. Cole, G. Comezaña, S. Costenoble, A. D. Elmendorf, J. P. C. Greenlees, L. G. Lewis, Jr., R. J. Piacenza, G. Triantafillou, and S. Waner.

[21] J.P. May. Simplicial Objects in Algebraic Topology. D. Van Nostrand, 1967.

[22] J.E. McClure and J.H. Smith. A solution of Deligne's Hochschild cohomology conjecture. In Proceedings of the JAMI conference on Homotopy Theory, volume 293 of Contemp. Math., pages 153-193, 2002.

[23] J.E. McClure and J.H. Smith. Cosimplicial objects and little n-cubes. I. Amer. J. Math., 126, pages 1109 $-1153,2004$.

[24] R. Street. The formal theory of monads. J. Pure Appl. Algebra, 2:149-168, 1972.

[25] D. Tamarkin and B. Tsygan. Cyclic formality and index theorems. Lett. Math. Phys., 56:85-97, 2001.

Macquarie University, North Ryde, 2109, NSW, Australia

E-mail address: mbatanin@ics.mq.edu.au

Mathematical Institute of the Academy, Žitná 25, 11567 Prague 1, The Czech Republic

E-mail address: markl@math.cas.cz 